\title{
ANOMALIES IN STRING THEORY WITH D-BRANES*
}

\section{DANIEL S. FREED ${ }^{\dagger}$ AND EDWARD WITTEN ${ }^{\ddagger}$}

Introduction. This paper is devoted to studying global anomalies in the worldsheet path integral of Type II superstring theory in the presence of $D$-branes. We will not consider orientifolds (or Type I superstrings) and so our string worldsheets will be oriented Riemann surfaces, mapped to a spacetime manifold $Y$, which is endowed with a spin structure since the model contains fermions. The first case to consider is that of closed worldsheets, without boundary. In this case, global anomalies cancel, and the worldsheet measure is globally well-defined, given that $Y$ is spin. This is the content of Corollary 4.7 of the present paper; for $Y=\mathbb{R}^{10}$ one has (Theorem 4.8) the further result, related to conformal invariance, that the global anomaly vanishes separately for left- and right-moving degrees of freedom. By contrast, for heterotic strings, global anomaly cancellation gives a restriction that is not evident from the point of view of the low energy effective field theory [W2,F2].

But we will find that Type II global worldsheet anomalies give some novel results when $D$-branes are present. Thus, we assume the existence of an oriented submanifold $Q$ of spacetime on which strings can end. We then consider a string worldsheet consisting of an oriented surface $\Sigma$ that is mapped to $Y$ with $\partial \Sigma$ mapped to $Q$. On $Q$, there is a field $A$ that is conventionally regarded as a $U(1)$ gauge field, though as we will explain this is not the correct interpretation globally. Another important part of the story is the Neveu-Schwarz $B$-field, which propagates in the bulk of spacetime. Assume for simplicity that $B=0$. In this case, the worldsheet measure contains two interesting factors which we write as follows:

$$
\operatorname{pfaff}(D) \cdot \exp \left(i \oint_{\partial \Sigma} A\right)
$$

Here pfaff $(D)$ is the pfaffian (or square root of the determinant) of the worldsheet Dirac operator $D$. The second factor is, in the conventional interpretation, the holonomy of $A$ around the boundary of $\Sigma$.

Our main result, Theorem 5.6, computes the anomaly in pfaff $(D)$. Namely, $\operatorname{pfaff}(D)$ is naturally not a function, but rather a section of a line bundle over the space of parameters - the space of maps of the worldsheet into spacetime and worldsheet metrics. This bundle carries a natural metric and connection, and the anomaly is the obstruction to the existence of a global flat section of unit norm. We will show that this connection is flat, but there is holonomy \pm 1 determined by the second Stiefel-Whitney class $w_{2}(\nu)$ of the normal bundle $\nu$ to the $D$-brane $Q$ in spacetime. ${ }^{1}$

This means that for $(1)$ to be well-defined (for $B=0$ ) there must be a compensating anomaly in the $A$-field. If $W_{3}(\nu)=0$, then this is achieved by interpreting $A$ as a $\operatorname{Spin}^{c}$ connection rather than an ordinary $U(1)$ gauge field. When $W_{3}(\nu) \neq 0$ (and

* Received July 28, 1999; accepted for publication January 30, 2000.

$\dagger$ Department of Mathematics, University of Texas, Austin, TX 78712, USA (dafr@math.utexas. edu). The research of this author is supported by NSF grant DMS-9626698.

$\ddagger$ Institute for Advanced Study, Olden Lane, Princeton NJ, 08540, USA (witten@sns.ias.edu). The research of this author is supported by NSF grant PHY-95-13835.

1 This problem exemplifies the difference between the topological anomaly and the geometric anomaly: the topological isomorphism class of this line bundle is determined by the third StiefelWhitney class $W_{3}(\nu)$, and this may vanish even if the holonomy is nontrivial. 
$B$ is topologically trivial) the anomaly rules out $Q$ as a possible $D$-brane in the theory. This result from string perturbation theory matches the nonperturbative description of $D$-brane charge as an element of $K$-theory [MM,W4]: the normal bundle $\nu$ must have $W_{3}(\nu)=0$ in order to define the charge. If $B \neq 0$ there is another term in (1) and a correspondingly more complicated interpretation of the $A$-field, leading to a generalization of the condition $W_{3}(\nu)=0$ which is stated in equations (1.12) and (6.9). Also, when $B \neq 0, D$-brane charge takes values in a twisted form of $K$-theory, as explained in section 5.3 of [W4]. The net effect is always that the $Q$ 's that make sense in perturbative string theory are the ones that have Thom classes in the appropriate $K$-group.

Therefore, our major tasks are to prove the anomaly formula and to properly interpret the $A$ - and $B$-fields. Since the results here are of direct interest in string theory, we begin in section 1 by explaining the physical implications of the anomaly. Here we analyze the $B \neq 0$ case as well as the simpler case when $B$ vanishes. We give examples to show that the anomaly can occur, and we also show how it relates to $D$-brane charge. Section 2 contains a heuristic argument for the anomaly in terms perhaps more palatable to physicists than the proofs which follow. From a mathematical point of view the proof, which is contained in section 5, combines ideas from topological index theory (1960s) and geometric index theory (1980s). The pertinent background material is quickly summarized in section 3 . The analysis of anomalies for closed strings is contained in section 4; some of those results are needed in section 5 as well. Section 6 is a commentary, in mathematical language, on some of the issues raised in the main part of the paper. First, we give a more precise description of the $A$ - and $B$-fields in terms of Čech theory. (A general mathematical framework which would apply to all occurrences of $p$-form fields in quantum field theory, string theory, and $M$-theory is still lacking, so we settle for the Čech description.) Second, we remark on some general features of Dirac operators on manifolds with boundary where the boundary conditions are local.

With great pleasure we dedicate this paper to Michael Atiyah. His influence is evident in every section. Not only did he (and his collaborators) develop topological $K$-theory and topological index theory, which are used here to compute a subtle sign whose definition is analytic, but he was also a pioneer in the application of these ideas to anomalies and to other problems in quantum field theory. Thus we hope that the mix of mathematics and theoretical physics in this paper is an appropriate tribute to him.

1. Role Of The Anomaly In String Theory. We consider the Type II superstring theory on a spacetime $Y$, beginning with the case that $B=0$. Recall that $Y$ is an oriented spin manifold. The $D$-brane is an oriented submanifold $Q \subset Y$. ( $Q$ is oriented because in Type II superstring theory, $D$-brane worldvolumes, being sources of Ramond-Ramond flux, must be oriented.) The string worldsheet $\Sigma$ is endowed with spin structures $\alpha$ and $\beta$ for the left and right-movers. We consider maps of $\Sigma$ into $Y$ which send the boundary $\partial \Sigma$ to $Q$. Our goal is to assess the well-definedness of the worldsheet path integral. The relevant factors are

$$
\operatorname{pfaff}(D) \cdot \exp \left(i \oint_{\partial \Sigma} A\right)
$$

(where $\operatorname{pfaff}(D)$ is the Pfaffian of the world-sheet Dirac operator $D$ and $A$ is the " $U(1)$ gauge field" on $Q$ ). Type II global anomaly cancellation for closed surfaces (applied 
to the double of $\Sigma$, which is closed) shows that the result does not depend on the spin structures (see Theorem 4.6). So we may as well assume that $\beta=\alpha$. Once this is done, the Dirac operator becomes real. So $\operatorname{pfaff}(D)$ is real. Its absolute value is well-defined, for example by zeta function or Pauli-Villars regularization. However, there is in general no natural way to define the sign of $\operatorname{pfaff}(D)$ as a number. We pick a particular sign, and proceed to see if we will run into a contradiction. For this, we consider a one-parameter family of $\Sigma$ 's, parametrized by a circle $C$. Thus, altogether we have a map $\phi: \Sigma \times C \rightarrow Y$, with $\phi(\partial \Sigma \times C) \subset Q$. The question is now whether, when one goes around the loop $C, \operatorname{pfaff}(D)$ comes back to itself or changes sign. Our main result (Theorem 5.6) is that under going around $C$,

$$
\operatorname{pfaff}(D) \rightarrow(-1)^{\alpha} \operatorname{pfaff}(D) .
$$

with

$$
\alpha=\left(\partial \Sigma \times C, \phi^{*}\left(w_{2}(Q)\right)\right) .
$$

Here $w_{2}(Q)$ is the second Stiefel-Whitney class of $Q$. If $\phi: \partial \Sigma \times C \rightarrow Q$ is an embedding, we can just write

$$
\alpha=\int_{\partial \Sigma \times C} w_{2}(Q)=\left(\partial \Sigma \times C, w_{2}(Q)\right) .
$$

In particular, if $w_{2}(Q)$ is non-zero, ${ }^{2} \operatorname{pfaff}(D)$ is not well-defined by as a number.

Let $\nu$ be the normal bundle to $Q$ in $Y$. Because $Y$ is $\operatorname{spin}\left(w_{1}(Y)=w_{2}(Y)=0\right)$ and $Q$ is oriented $\left(w_{1}(Q)=0\right)$, the Whitney sum formula

$$
\left(1+w_{1}(Y)+w_{2}(Y)+\ldots\right)=\left(1+w_{1}(Q)+w_{2}(Q)+\ldots\right)\left(1+w_{1}(\nu)+w_{2}(\nu)+\ldots\right)
$$

gives $w_{1}(\nu)=0$ and $w_{2}(\nu)=w_{2}(Q)$. Hence we can restate the above anomaly formulas, for example

$$
\alpha=\left(\partial \Sigma \times C, \phi^{*}\left(w_{2}(\nu)\right)\right) .
$$

This formulation turns out to be more natural in $K$-theory.

When $\operatorname{pfaff}(D)$ is not well-defined, the string theory is well-defined only if the second factor in (1.1) has precisely the same ambiguity. In other words, $A$ must not be globally a conventional $U(1)$ gauge field, for which the holonomy around a loop is well-defined as an element of $U(1)$. Rather, the holonomy around $\partial \Sigma$

$$
\exp \left(i \oint_{\partial \Sigma} A\right)
$$

must be well-defined only up to multiplication by \pm 1 , and must change sign whenever $\operatorname{pfaff}(D)$ does.

There is another important differential-geometric object that has the same sign ambiguity. Let $\omega$ be the Levi-Civita connection of the manifold $Q$. Its structure group is $S O(n), n$ being the dimension of $Q$. Consider the trace of the holonomy

\footnotetext{
${ }^{2}$ And can be detected by a map from $\partial \Sigma \times C$, which is a two-torus. By analogy with many other problems involving global anomalies, we expect, though we will not try to prove here, that if $w_{2}(Q)$ is nonzero but can only be detected by a map from a surface of higher genus, then the same consequences will follow upon analyzing the factorization of the string measure when $\Sigma$ breaks into pieces, or in other words by analyzing unitarity of string scattering amplitudes.
} 
of this connection in the spin representation $S$ of the double cover $\operatorname{Spin}(n)$. This is customarily denoted

$$
\operatorname{Tr} P \exp \left(\oint_{\partial \Sigma} \omega\right)
$$

This holonomy is well-defined only up to sign (because there are two ways to lift an element of $S O(n)$ to $S \operatorname{pin}(n))$. In going around a one-parameter family of loops, parametrized by a circle $C$, the holonomy (1.3) is multiplied by exactly the same sign factor that appears in (1.2). The upshot, then, is that if $A$ is the right kind of geometrical object to make the worldsheet string measure well-defined, then the product

$$
\operatorname{Tr} P \exp \left(\oint_{\partial \Sigma} \omega\right) \cdot \exp \left(i \oint_{\partial \Sigma} A\right)
$$

is likewise well-defined.

What appears in (1.4) is the trace of the holonomy in going around the loop $\partial \Sigma$, not for ordinary spinors on $Q$, but for spinors of charge 1 with respect to $A$. Such charged spinors are sections not of $S(Q)$, the "spin bundle" of $Q$, but of $S(Q) \otimes \mathcal{L}$, where $\mathcal{L}$ is the "line bundle" on which $A$ is a connection. The meaning of the global anomaly (for topologically trivial $B$-field) is thus that the globally defined object is not in general $S(Q)$ or $\mathcal{L}$, but the tensor product $S(Q) \otimes \mathcal{L}$.

Such a tensor product defines a so-called "Spin ${ }^{c}$ structure" of $Q$. The global anomaly thus implies that (again, for trivial $B$-field) $Q$ must be $\operatorname{Spin}^{c}$, and more specifically a $\operatorname{Spin}^{c}$ structure can be constructed from the physical data, namely from the Levi-Civita connection $\omega$ and the field $A$.

Since, as we have seen above, $w_{2}(Q)=w_{2}(\nu)$, it is equivalent to endow $Q$ with a $\operatorname{Spin}^{c}$ structure or to endow its normal bundle with such a structure. In other words, being given a bundle $S(Q) \otimes \mathcal{L}$ determines a bundle $S(\nu) \otimes \mathcal{L}$, where $S(\nu)$ are the spinors of the normal bundle.

\section{Physical Interpretation}

The phenomenon just indicated was first encountered by hand in a very special case [W3], but it has a theoretical interpretation that we will now recall.

Naively speaking, the conserved charges associated with wrapping of $D$-branes in a spacetime manifold $Y$ take values in $H^{*}(Y ; \mathbb{Z})$, the cohomology of $Y$. A closer look shows, however [MM,W4], that, when the $B$-field is topologically trivial, $D$-brane charge takes values in $K$-theory, in fact in $K(Y)$ or $K^{1}(Y)$ for Type IIB or Type IIA string theory.

However, for a $D$-brane wrapped on $Q$ to define a class in $K(Y)$ (or $K^{1}(Y)$ ), its normal bundle $\nu$ must be endowed with a $\operatorname{Spin}^{c}$ structure. This results from a standard construction of Atiyah, Bott, and Shapiro; see [W4], section 4.3 for an explanation in the context of the application to string theory. Hence, if the $K$-theory interpretation of $D$-branes is correct, Type II $D$-branes (at $B=0$ ) must be naturally endowed with a Spin ${ }^{c}$ structure on the normal bundle. Equivalently, since $w_{2}(Q)=w_{2}(\nu)$, the Type II $D$-brane world-volume $Q$ must carry a $\operatorname{Spin}^{c}$ structure. As we have just explained, the global anomaly formula (1.2) has exactly this consequence; because of this anomaly, a $D$-brane is endowed not with a $U(1)$ gauge field, as it naively appears, but with a Spin $^{c}$ structure. 
Let us now analyze the conditions under which $Q$ admits a $\operatorname{Spin}^{c}$ structure. Consider the exact sequence of abelian groups

$$
0 \rightarrow \mathbb{Z} \stackrel{2}{\longrightarrow} \mathbb{Z} \stackrel{r}{\longrightarrow} \mathbb{Z}_{2} \rightarrow 0
$$

with the first map being multiplication by 2 and the second reduction modulo 2 . This short exact sequence leads to a long exact cohomology sequence:

$$
\ldots H^{2}(Q ; \mathbb{Z}) \stackrel{r}{\longrightarrow} H^{2}\left(Q ; \mathbb{Z}_{2}\right) \stackrel{\beta}{\longrightarrow} H^{3}(Q ; \mathbb{Z}) \rightarrow \ldots
$$

The object $\beta\left(w_{2}(Q)\right) \in H^{3}(Q ; \mathbb{Z})$ is the third Stiefel-Whitney class $W_{3}(Q)$.

Vanishing of $W_{3}(Q)$ is equivalent to $Q$ admitting $\operatorname{Spin}^{c}$ structure. Indeed, exactness of $(1.6)$ says that $w_{2}(Q)=r(x)$ for some $x \in H^{2}(Q ; \mathbb{Z})$ if and only if $W_{3}(Q)=0$. When there is no two-torsion in $H^{2}(Q ; \mathbb{Z})$, there is precisely one Spin ${ }^{c}$ structure on $Q$ for every such $x$. In fact, given a $\operatorname{Spin}^{c}$ connection $\omega+A$, if $\mathcal{L}$ is the "line bundle" on which $A$ is a connection, then $\mathcal{M}=\mathcal{L}^{2}$ is an honest line bundle, and $x=c_{1}(\mathcal{M})$ obeys $r(x)=w_{2}(Q)$. Conversely, given $x$ with $r(x)=w_{2}(Q)$, we let $\mathcal{M}$ be a complex line bundle with $c_{1}(\mathcal{M})=x$, and we let $A$ be a connection on the "line bundle" $\mathcal{L}=\mathcal{M}^{1 / 2}$. (When there is two-torsion in $H^{2}(Q ; \mathbb{Z})$, there are different square roots of $\mathcal{M}$ and hence more than one $\operatorname{Spin}^{c}$ structure for given $x$.)

\section{Inclusion Of B-Field}

Now let us discuss what to do when $W_{3}(Q) \neq 0$, so that the anomaly cannot be cancelled by picking a $\operatorname{Spin}^{c}$ structure on $Q$. So far we have assumed that the $B$-field is topologically trivial, in which case it can simply be ignored in analyzing the anomalies. But now we must include it. The $B$-field couples to the world-sheet $\Sigma$ in bulk, and in its presence an additional term must be added to (1.4), which now becomes

$$
\operatorname{pfaff}(D) \exp \left(i \oint_{\partial \Sigma} A+i \int_{\Sigma} B\right)
$$

This expression has the gauge invariance

$$
A \rightarrow A-\Lambda, B \rightarrow B+d \Lambda,
$$

where $\Lambda$ is any connection on an arbitrary complex line bundle $\mathcal{M}$.

Let us discuss, in stages, the meaning of (1.7). First we consider the $B$-field in the theory of closed oriented bosonic strings. In this theory, for a closed surface $\Sigma$, the $B$-field gives a phase

$$
W(\Sigma ; B)=\exp \left(i \int_{\Sigma} B\right) .
$$

This is a complex number of modulus one, an element of $U(1)$; we can think of it as the holonomy of $B$ over $\Sigma$. On the other hand, if $\Sigma$ has a boundary, then $W(\Sigma ; B)$ is not gauge-invariant. It must be regarded as an element of a complex line $\mathcal{L}_{B}$ associated to $\partial \Sigma$ by $B$. The line $\mathcal{L}_{B}$ depends on $\partial \Sigma$, but we do not show this in the notation. For example, if $\partial \Sigma$ is a single circle, then $\mathcal{L}_{B}$ varies as $\partial \Sigma$ varies to give a complex line bundle over the loop space of $Y$ (or over a component of this loop space determined by the homotopy class of $\Sigma$ ). We write $L Y$ for the loop space of $Y$.

The interpretation of $W(\Sigma ; B)$ as taking values in a complex line $\mathcal{L}_{B}$ may seem slightly abstract, but it actually reflects an idea that is familiar to physicists. The 
two-form field $B$ in spacetime determines (by integration over the loop) a one-form field or abelian gauge field on loop space; this abelian gauge field is a connection on a complex line bundle $\mathcal{L}_{B}$ over $L Y$.

Now we introduce in the bosonic string a $D$-brane with world-volume $Q$. We require $\partial \Sigma \subset Q$. In this situation, there is a completely gauge-invariant extension of (1.9), namely

$$
W(\Sigma ; B, A)=\exp \left(i \oint_{\partial \Sigma} A+i \int_{\Sigma} B\right)
$$

We interpret this expression to mean that $A$ gives a trivialization of the restriction of $\mathcal{L}_{B}$ to loops that lie in $Q$. In other words, $A$ trivializes the restriction of $\mathcal{L}_{B}$ to $L Q$, the loop space of $Q$; when $\partial \Sigma \subset Q, W(\Sigma ; B, A)$ is a gauge-invariant version of $W(\Sigma ; B)$.

Now, let us consider the superstring and the problem of defining the product

$$
\operatorname{pfaff}(D) \cdot \exp \left(i \int_{\Sigma} B\right) \cdot \exp \left(i \oint_{\partial \Sigma} A\right) .
$$

For simplicity of exposition in what follows, we suppose that the boundary of $\Sigma$ is a single circle; the generalization is evident. In the mathematical theory, $\operatorname{pfaff}(D)$, though not well-defined as a number, is defined as a section of a Pfaffian line bundle Pfaff over $L Q$. As we have already discussed, the second factor in (1.11) must likewise be interpreted as a section of a line bundle $\mathcal{L}_{B}$. We also have just explained that, in the bosonic string theory, the last factor in (1.11) should be interpreted as trivializing the restriction of $\mathcal{L}_{B}$ to $L Q$. For the superstring, since there is an extra factor in (1.11), the interpretation is different. The last factor in (1.11) must in this case be understood as giving a trivialization of Pfaff $\otimes \mathcal{L}_{B}$, restricted to $L Q$.

This means in particular that for the bosonic string, the restriction of $B$ to $Q$ must be topologically trivial. For the superstring, the restriction of $B$ to $Q$ is in general topologically nontrivial, but its topological type is uniquely determined, by the fact that Pfaff $\otimes \mathcal{L}_{B}$ (restricted to loops in $Q$ ) must be topologically trivial.

\section{Topological Classification Of B-Fields}

To make this somewhat more explicit, we recall the topological classification of $B$ fields. Topologically, $B$-fields on $Y$ are classified by a characteristic class $\zeta \in H^{3}(Y ; \mathbb{Z})$. At the level of differential forms, $\zeta$ is represented by $H / 2 \pi$, where $H=d B$ is the curvature of $B$. By integrating $\zeta$ over a loop, we get a two-dimensional characteristic class on $L Y$, which in fact equals $c_{1}\left(\mathcal{L}_{B}\right)$. Flat $B$-fields, that is, $B$-fields whose field strength $H$ vanishes, are classified by the holonomy around closed surfaces, which gives a cohomology class in $H^{2}(Y ; \mathbb{R} / \mathbb{Z})$. A flat $B$-field has a torsion characteristic class $\zeta$ computed by the "Bockstein" $\operatorname{map} \beta: H^{2}(Y ; \mathbb{R} / \mathbb{Z}) \rightarrow H^{3}(Y ; \mathbb{Z})$. (A similar $\beta$ appears in (1.6).) Notice by comparison the analogous classification of abelian gauge fields, where the degree is shifted down by one.

Pfaff $\otimes \mathcal{L}_{B}$ is trivial if ${ }^{3}$

$$
\left.\zeta\right|_{Q}=W_{3}(Q)
$$

\footnotetext{
${ }^{3}$ In fact, (1.12) is only a sufficient condition, but is the only cohomological condition that implies triviality of Pfaff $\otimes \mathcal{L}_{B}$. As in footnote 2 at the beginning of section 1 , we expect that factorization and unitarity will lead to the full requirement of $(1.12)$.
} 
and we claim that this is the right topological condition on the $B$-field. The integral of the left hand side over $\partial \Sigma$ is the first Chern class of $\mathcal{L}_{B}$. The holonomy of Pfaff was stated in (1.2), and this may be reinterpreted to say that the class of Pfaff as a flat line bundle in $H^{1}(L Q ; \mathbb{R} / \mathbb{Z})$ is the integral of $w_{2}(Q)$ over $\partial \Sigma$. Using the remark following (1.6) and the fact that the Bockstein commutes with integration, we see that the first Chern class of Pfaff is the integral of $W_{3}(Q)$ over $\partial \Sigma$.

In particular, $B$ restricted to $Q$ is topologically trivial if and only if $W_{3}(Q)=0$, or in other words (given what we have seen above) if and only if $Q$ is $\operatorname{Spin}^{c}$.

To be explicit, we will give an example of a $B$-field on $Q$ (or on any spacetime $Y$ over which $w_{2}(Q)$ extends) with $\zeta=W_{3}(Q)$. Just as an abelian gauge field is completely determined up to isomorphism by its holonomy around closed loops, a $B$ field is completely determined up to isomorphism by its holonomy over closed surfaces. Since $W_{3}(Q)=\beta\left(w_{2}(Q)\right)$ is a torsion class, there exists a flat $B$-field, whose holonomy over a closed surface $\Sigma$ depends only on the homology class of $\Sigma$, with $\zeta=W_{3}(Q)$. Such a flat $B$-field is indeed described by the elegant formula

$$
W(\Sigma ; B)=(-1)^{\left(\Sigma, w_{2}(Q)\right)} .
$$

In other words, the isomorphism class of this flat $B$-field is the image of $w_{2}(Q)$ under $H^{2}\left(Q ; \mathbb{Z}_{2}\right) \rightarrow H^{2}(Q ; \mathbb{R} / \mathbb{Z})$. Its characteristic class $\zeta$ is then computed by the Bockstein map to be $W_{3}(Q)$.

\section{Examples}

We will give a few concrete examples to which the discussion applies.

Every oriented manifold of dimension $\leq 3$ is spin. A simple example of a fourmanifold that is not spin is $Q=\mathbb{C} P^{2}$. Then $H^{2}(Q ; \mathbb{Z})=\mathbb{Z}$, and $H^{2}\left(Q ; \mathbb{Z}_{2}\right)=\mathbb{Z}_{2}$. The second Stiefel-Whitney class $w_{2}(Q)$ is the nonzero element of $H^{2}\left(Q ; \mathbb{Z}_{2}\right)$. Since $H^{3}(Q ; \mathbb{Z})=0$, we have $W_{3}(Q)=0$ and $Q$ is $\operatorname{Spin}^{c}$. In fact, the map $r: H^{2}(Q ; \mathbb{Z}) \rightarrow$ $H^{2}\left(Q ; \mathbb{Z}_{2}\right)$ is just reduction modulo 2 ; the elements $x \in H^{2}(Q ; \mathbb{Z})$ with $r(x)=w_{2}(Q)$ correspond to the odd integers in $H^{2}(Q ; \mathbb{Z}) \cong \mathbb{Z}$.

If a $D$-brane has world-volume $Q=\mathbb{C} P^{2}$, then (assuming that the $B$-field vanishes) the global anomaly means that the " $U(1)$ gauge field" $A$ does not obey standard Dirac quantization. Rather, $A$ is a connection on a "line bundle" whose square has an odd first Chern class $x$ (congruent to $w_{2}(Q) \bmod 2$ ). If $L \subset Q$ is a generator of $H_{2}(Q ; \mathbb{Z})$, then we have $\left(L, w_{2}(Q)\right) \neq 0$ and hence

$$
\int_{L} \frac{F_{A}}{2 \pi}=\frac{x}{2}
$$

with $x$ an odd integer. In particular, $x$ cannot be zero.

Every oriented manifold of dimension $\leq 4$ is $\operatorname{Spin}^{c}$. To give a simple example of a five-manifold that is not $\operatorname{Spin}^{c}$, let $Q^{\prime}=\bar{Q} \tilde{\times} \mathbb{S}^{1}$ be a $\mathbb{C} P^{2}$ bundle over $\mathbb{S}^{1}$ in which, as one goes around the $\mathbb{S}^{1}$, the fiber $\mathbb{C} P^{2}$ undergoes complex conjugation. Complex conjugation acts on $H^{2}(Q ; \mathbb{Z})$ by multiplication by -1 . $Q^{\prime}$ is not a $\operatorname{Spin}^{c}$ manifold, for the following reason. An $\tilde{x} \in H^{2}\left(Q^{\prime} ; \mathbb{Z}\right)$ obeying $r(\tilde{x})=w_{2}\left(Q^{\prime}\right)$ would have to reduce on each fiber of the projection $Q^{\prime} \rightarrow \mathbb{S}^{1}$ to an odd element $x \in H^{2}(Q ; \mathbb{Z}) \cong \mathbb{Z}$. Because of the monodromy, $x$ would have to change sign in going around the $\mathbb{S}^{1}$, which is impossible. So $Q^{\prime}$ is not $\operatorname{Spin}^{c}$.

To illustrate the ideas of the present paper requires considering topologically non-trivial $D$-brane world-volumes such as $Q$ or $Q^{\prime}$. But spacetime itself can be very simple, for example $Y=\mathbb{R}^{10}$. Since $Y$ is contractible, the $B$-field is automatically 
topologically trivial and can be ignored. We will give examples of $D$-brane states in $\mathbb{R}^{10}$ which are or are not allowed.

On general grounds, any manifold of dimension $\leq 5$ can be embedded in $\mathbb{R}^{10}$. For completeness, we will describe a simple embedding of $Q$ and $Q^{\prime}$. Identify $Q=\mathbb{C} P^{2}$ with the space of vectors $\psi$ of unit norm in $\mathbb{C}^{3}$, modulo phase rotations. Let $\lambda_{a}$ be a suitably normalized basis of the Lie algebra of $S U(3)$, and

$$
\phi_{a}=\left\langle\psi\left|\lambda_{a}\right| \psi\right\rangle
$$

Then $\sum_{a=1}^{8} \phi_{a}^{2}=1$, so using the $\phi_{a}$ as coordinates, we get an embedding of $\mathbb{C} P^{2}$ in $\mathbb{S}^{7}$, which obviously embeds in $\mathbb{R}^{10}$. To embed $Q^{\prime}$ in $\mathbb{R}^{10}$, first note that $\mathbb{S}^{8} \times \mathbb{S}^{1}$ can be embedded in $\mathbb{R}^{10}$ (for instance as the set of points a distance $\epsilon$ from a circle $\mathbb{S}^{1} \subset \mathbb{R}^{10}$, for suitably small $\epsilon$ ). Hence it suffices to embed $Q^{\prime}$ in $\mathbb{S}^{8} \times \mathbb{S}^{1}$. For this, we first add one more coordinate $y$ to the $\phi_{a}$, to get a copy of $\mathbb{S}^{8}$ defined by $\sum_{a} \phi_{a}^{2}+y^{2}=1$, and embed $Q$ in $\mathbb{S}^{8}$ as the subset with $y=0$ and $\phi_{a}$ as before. Now, complex conjugation of $\mathbb{C} P^{2}$ acts on the set of eight $\phi_{a}$ with determinant -1 (three eigenvalues +1 and five -1 ), so if we take it to act on $y$ as multiplication by -1 , we get an element $T \in S O(9)$. $S O(9)$ is a connected group of symmetries of $\mathbb{S}^{8}$. Let $R(\theta), 0 \leq \theta \leq 2 \pi$ be a path in $S O(9)$ with $R(0)=1$ and $R(2 \pi)=T$. Finally, embed $Q^{\prime}$ in $\mathbb{S}^{8} \times \mathbb{S}^{1}$ by mapping $(P, \theta)$ (with $P \in \mathbb{C} P^{2} \subset \mathbb{S}^{8}$ and $\theta \in \mathbb{S}^{1}$ ) to $(R(\theta) P, \theta) \in \mathbb{S}^{8} \times \mathbb{S}^{1}$.

Now, given a $D$-brane in $\mathbb{R}^{10}$ with world-volume $Q$ or $Q^{\prime}$, we will explain how to construct a family of string worldsheets that detects the global anomaly. We take $\Sigma$ to be a disc; its boundary is a circle $\partial \Sigma$. Letting $C$ be another circle, the boundary of $\Sigma \times C$ is $W=\partial \Sigma \times C$, a two-torus. For a generator of $H_{2}(Q ; \mathbb{Z})$ or $H_{2}\left(Q^{\prime} ; \mathbb{Z}\right)$ we can take a two-sphere $L$. We pick a degree one map $\phi_{0}: W \rightarrow L$; then, since $\mathbb{R}^{10}$ is contractible, one can extend $\phi_{0}$ to a map $\phi: \Sigma \times C \rightarrow \mathbb{R}^{10}$. This gives a relatively simple example of a family of worldsheets for which there is a global anomaly. Hence, the $D$-brane $Q^{\prime} \subset \mathbb{R}^{10}$ is not allowed, and the $D$-brane $Q \subset \mathbb{R}^{10}$ is allowed but must support a " $U(1)$ gauge field" with half-integral flux.

\section{Conservation Laws And The Anomaly}

By further discussion of $D$-branes in $\mathbb{R}^{10}$, we can show the relation of the anomaly to $D$-brane charges.

We work in Lorentz signature, and split $\mathbb{R}^{10}$ as $\mathbb{R} \times \mathbb{R}^{9}$, where $\mathbb{R}$ parametrizes "time" and $\mathbb{R}^{9}$ is "space." We consider a $D$-brane whose world-volume near time zero is approximately $Q=\mathbb{R} \times Q_{0}$, with $Q_{0} \subset \mathbb{R}^{9}$. We will focus on Type IIA superstrings, so $Q_{0}$ is of even dimension. To detect the anomaly, the dimension is at least four.

The world-volume of the $D$-brane will not look like $\mathbb{R} \times Q_{0}$ for all time. The $D$-brane will oscillate, emit radiation, and contract. In the far future, it will decay to a final state consisting of outgoing stable particles. The only known stable particles in Type IIA superstring theory in $\mathbb{R}^{10}$ are massless particles (the graviton and its superpartners) and $D 0$-branes, together with the familiar multi- $D 0$-brane bound states. (There are no conserved charges for higher branes because $\mathbb{R}^{10}$ is contractible.) If there are no additional stable particles, then our initial state will decay to an assortment of the known ones.

If so, we can predict how many net $D 0$-branes will be produced - that is the difference between the number of $D 0$-branes and anti- $D 0$-branes in the final state. It 
must equal the $D 0$-brane charge of the initial state, which [GHM,CY,MM] is

$$
N_{0}=\int_{Q_{0}} \sqrt{\hat{A}(Q)} \frac{1}{\sqrt{\hat{A}(\nu)}} \exp \left(c_{1}(\mathcal{L})\right),
$$

where $\hat{A}$ is the total A-roof class, and $c_{1}(\mathcal{L})$ is the first Chern class of the "complex line bundle" $\mathcal{L}$ on which the "U(1) gauge field" $A$ of the $D$-brane is a connection. Now, using the fact that the tangent bundle of $\mathbb{R}^{10}$ is trivial, and splits as $T Q \oplus \nu$ (with $T Q$ the tangent bundle to $Q$ ), we have $\hat{A}(\nu)=\hat{A}(Q)^{-1}$. So we rewrite the formula for the total $D 0$-brane charge as

$$
N_{0}=\int_{Q_{0}} \hat{A}\left(Q_{0}\right) \exp \left(c_{1}(\mathcal{L})\right) .
$$

(We have written here $\hat{A}\left(Q_{0}\right)$ rather that $\hat{A}(Q)$; the two are equal as $T Q=T Q_{0} \oplus \epsilon$, where $\epsilon$ is a trivial real line bundle that incorporates the "time" direction.)

Now, if $\mathcal{L}$ were a complex line bundle, then in general $N_{0}$ would not be an integer. For example, for $Q_{0}=\mathbb{C} P^{2}$ and $\mathcal{L}$ trivial, we would have $N_{0}= \pm 1 / 8$ (depending on orientation). When $N_{0}$ is not integral, the initial $D$-brane state cannot decay to known stable particles.

We either must postulate the existence of new conservation laws for Type IIA strings in $\mathbb{R}^{10}$ or achieve integrality of $N_{0}$ by some other modification of the rules. As we have seen, the global anomaly means that $A$ is not a $U(1)$ connection, but rather determines a $\operatorname{Spin}^{c}$ structure $S(Q) \otimes \mathcal{L}$ on $Q$ or equivalently a $\operatorname{Spin}^{c}$ structure $S\left(Q_{0}\right) \otimes \mathcal{L}$ on $Q_{0}$. With this interpretation of $A$ and $\mathcal{L}$, the Atiyah-Singer index theorem states that the right hand side of (1.14) is the index of the Dirac operator (for spinors on $Q_{0}$ valued in $\left.S\left(Q_{0}\right) \otimes \mathcal{L}\right)$, so that $N_{0}$ is always integral.

Thus, the anomaly enables us to avoid having to postulate new conservation laws for $D$-branes in $\mathbb{R}^{10}$.

2. Qualitative Explanation Of Anomaly. To give a qualitative explanation of the anomaly, we consider a $D p$-brane with oriented world-volume $Q$ in a spacetime $Y$. If the given $D$-brane is the only one in spacetime, then a consideration of the string spectrum will not lead in an obvious way to a result involving $w_{2}(Q)$. In fact, if there is only one $D$-brane, the only open strings are the $p$ - $p$ open strings with both ends on $Q$. The ground state of the $p-p$ open strings in the Ramond sector consists of sections of $S(Q) \otimes S(\nu)$ where $S(Q)$ is the bundle of spinors on $Q$, and $S(\nu)$ is the bundle of spinors on the normal bundle $\nu$ to $Q$ in $Y$. This tensor product exists whether $Q$ is spin or not (given only that $Y$ is spin), so merely by quantizing the $p-p$ open strings, we get no condition involving $w_{2}(Q)$. To obtain such a condition, we will study global worldsheet anomalies, as explained in the introduction.

Suppose, however, that an additional pair of $D$-branes, consisting of a space-filling 9- $\overline{9}$ pair, is present. ${ }^{4}$ We suppose that the gauge fields on the 9 -brane and $\overline{9}$-brane are trivial. In the presence of the additional branes, there are additional open strings such as the 9- $p$ open strings. The ground state Ramond sector 9- $p$ strings are sections of $S(Q) \otimes \mathcal{L}$, where again $S(Q)$ are the spinors on $Q$ and $\mathcal{L}$ is the "line bundle" on which the " $U(1)$ gauge field" $A$ on $Q$ is a connection.

\footnotetext{
${ }^{4}$ For Type IIB, these can be ordinary supersymmetric branes. For Type IIA, we could in this argument use instead the nonsupersymmetric 9-brane considered in $[\mathrm{H}]$.
} 
So the tensor product $S(Q) \otimes \mathcal{L}$ must exist, and we learn what was promised in the introduction: $Q$ must be $\operatorname{Spin}^{c}$, and the "gauge field" on $Q$ really defines a $\operatorname{Spin}^{c}$ structure.

In our actual problem, such additional branes are absent. We do not want to assume continuous creation and annihilation of brane-antibrane pairs along the lines of [S], for this involves somewhat speculative physics. If one is willing to make such assumptions, the relation of branes to $K$-theory and the $\operatorname{Spin}^{c}$ character of $Q$ can indeed be deduced, as in [W4]. Our intent here is to show that the requirement for $Q$ to carry a $\operatorname{Spin}^{c}$ structure can be deduced with only conservative assumptions about physics, by computing the global worldsheet anomaly.

Nevertheless, the fact that in the presence of a 9-brane, the quantization of the 9- $p$ open strings would make our result obvious is a starting point for a precise mathematical computation of the global anomaly. In essence, whether or not 9-branes can be continuously created in the physics, we can create them in the math, at least for the purposes of computing a global anomaly. This may be done as follows.

Consider any family of open string worldsheets $\Sigma$ with specified boundary conditions on the boundary components of $\Sigma$. The change in the global anomaly under a change in the boundary conditions is local, that is it only depends on the properties near the boundary of $\Sigma$. Such locality is perhaps more familiar for perturbative anomalies, which are expressed as integrals of differential forms. Global anomalies, however, also obey a suitable form of locality. They are computed topologically as "integrals" in $K$-theory, which obey the following excision property: If two $K$-theory classes agree outside an open set $U$, then the difference of their "integrals" can be computed on $U$. Geometrically, global anomalies are adiabatic limits of $\eta$-invariants, which also obey a factorization relation, though a more delicate one [DF]. These relations give the locality we want for the global anomaly.

Using this locality, we can reduce to a convenient set of boundary conditions. In fact, if one places 9-brane boundary conditions on all components of $\partial \Sigma$, there is no global anomaly. This statement is proved in Proposition 5.10, again using factorization. The statement is closely related to Type II global anomaly cancellation for closed surfaces without $D$-branes, because with 9-brane boundary conditions on all boundaries, the Dirac equation on the surface-with-boundary $\Sigma$ is equivalent to a chiral Dirac equation (acting on spinors of one chirality only) on the double of $\Sigma$.

Hence, the anomaly for the family $\Sigma \times C$ of open string world-sheets, with a map $\phi: \Sigma \times C \rightarrow Y$, depends only on the restriction of $\phi$ to $\partial \Sigma \times C$ and the boundary conditions on $\partial \Sigma \times C$. Once this is known, the anomaly can be evaluated by the following sleight of hand. We let $\Theta$ be an annulus. We select one distinguished component $\partial \Theta^{\prime}$ of the boundary of $\Theta$, and we select an isomorphism of $\partial \Theta^{\prime}$ to $\partial \Sigma$ (assuming $\partial \Sigma$ connected, in which case both of them are circles; the generalization to arbitrary $\partial \Sigma$ is clear). Then we pick a map $\tilde{\phi}: \Theta \times C \rightarrow Y$ which coincides with $\phi$ in a neighborhood of $\partial \Theta^{\prime} \times C$.

Now, we will compare the global anomalies for two different sets of Dirac operators that differ only by changes of boundary conditions:

(1) In the first case, we consider a Dirac operator on $\Sigma$ with $p$-brane boundary conditions, plus a Dirac operator on $\Theta$ with 9-brane boundary conditions at each boundary.

(2) In the second case, we consider a Dirac operator on $\Sigma$ with 9-brane boundary conditions, and a Dirac operator on $\Theta$ with $p$-brane boundary conditions on $\partial \Theta^{\prime}$ and 9-brane boundary conditions on the other boundary component. 
The total global anomaly in going around the loop $C$, summed over the two Dirac operators that are considered in each case, is the same in case (1) and case (2), because the union of all the boundaries and boundary maps are the same in the two cases. All we have done in going from case (1) to case (2) is to cut out neighborhoods of boundary components of $\Sigma \times C$ and $\Theta \times C$ and exchange them.

We also observe the following:

(I) In case (1), the global anomaly comes entirely from the Dirac operator on $\Sigma$, since the Dirac operator on $\Theta$ has 9-brane boundary conditions on each component. Hence, in case (1), the global anomaly is equal to what we want to calculate.

(II) In case (2), the global anomaly comes entirely from the Dirac operator on $\Theta$, since the Dirac operator on $\Sigma$ has 9-brane boundary conditions on each component.

In case (2), because $\Theta$ is an annulus with 9-brane boundary conditions at one end and $p$-brane boundary conditions at the other, it describes the propagation of 9- $p$ strings. As we remarked before, the ground states of such strings are sections of $S(Q) \otimes \mathcal{L}$ in the Ramond sector (or of $S(\nu) \otimes \mathcal{L}$ in the Neveu-Schwarz sector). Absence of global anomalies in their propagation is equivalent (as in the analysis of global anomalies in quantum mechanics in [W2]) to the existence of $S(Q) \otimes \mathcal{L}$ (or of $S(\nu) \otimes \mathcal{L})$

Thus, absence of the global anomaly for the Dirac operator on $\Sigma$ with $p$-brane boundary conditions is equivalent to existence of $S(Q) \otimes \mathcal{L}$, as we wished to show. A rigorous argument can be found in section 5 .

3. Anomalies and Index Theory. The path integral over a fermionic field $f$ is the regularized pfaffian, or ${ }^{5}$ determinant, of a Dirac operator $D$. It depends on the bosonic fields $b$ which couple to $f$, but rather than being a complex-valued function on the space $B$ of these bosons it is a section pfaff $D$ of a complex line bundle Pfaff $D \rightarrow B$. This section is part of the effective action we must integrate over $B$, and to do so we must find a global nonzero section 1: $B \rightarrow$ Pfaff $D$ and integrate instead the function pfaff $D / \mathbf{1}$. The anomaly is the obstruction to finding the trivializing section 1 . (More precisely, we must trivialize the product of the pfaffian line bundle and line bundles from other terms in the effective action, such as the additional contributions to (1.11).) This may be interpreted topologically, in which case the Atiyah-Singer index theorem is used to determine the topology of the pfaffian line bundle. More relevant to the physics is a geometric interpretation, in which we seek a flat section 1 of unit norm relative to the natural metric and connection on Pfaff $D$. (This distinction is important in our problem-compare Theorem 5.5 and Theorem 5.6.) For that we use differential geometric index theorems involving curvature forms and $\eta$-invariants. If the anomaly vanishes then $\mathbf{1}$, and so the effective action, is determined up to a phase on each connected component of $B$. See [F1], [F2] for more details. In this section we briefly summarize results from index theory-both topological and geometric - in the form we need.

Suppose $\pi: X \rightarrow Z$ is a fiber bundle whose fibers ${ }^{6} X / Z$ are closed manifolds endowed with a spin structure. Recall that $K O(X)$ is the group of virtual real vector

\footnotetext{
${ }^{5}$ In Minkowski space all fields are real and we can write the fermionic kinetic term as $\frac{1}{2} \psi \not D \psi$, so that the path integral over $\psi$ gives a real pfaffian. Often a pfaffian may be written as a determinant of a "smaller" Dirac operator. Since under Wick rotation the fermions are usually complexified, in Euclidean field theory the pfaffians and determinants are usually complex.

${ }^{6}$ Here $X / Z$ denotes the fiber of $X \rightarrow Z$. As usual, a spin structure on a manifold means a spin structure on its tangent bundle, here the tangent bundle $T(X / Z)$ along the fibers.
} 
bundles on $X$ up to equivalence, and $K O^{-n}(X) \subset K O\left(S^{n} \times X\right)$ is the subspace of isomorphism classes of virtual bundles trivial on $S^{n} \vee X$. The spin structure on the fibers determines a pushforward map

$$
\pi_{!}^{X / Z}: K O(X) \longrightarrow K O^{-n}(Z)
$$

where $n=\operatorname{dim} X / Z$. Given a real vector bundle $E \rightarrow X$, the family of Dirac operators on $X / Z$ coupled to $E$ has an index in $K O^{-n}(Z)$. The Atiyah-Singer index theorem [AS2] asserts that this analytic index equals $\pi_{!}^{X / Z}([E])$, where $[E] \in K O(X)$ is the isomorphism class of $E$. For Dirac operators coupled to complex bundles we have a similar picture with complex $K$-theory replacing real $K O$-theory. In general there is no cohomological formula for the integral or mod 2 characteristic classes of the index; over the rationals we can express the Chern character of the index (in $K$-theory) in terms of the Chern character of $E$ :

$$
\operatorname{ch} \pi_{!}^{X / Z}([E])=\pi_{*}^{X / Z}(\hat{A}(X / Z) \operatorname{ch}(E)),
$$

where $\pi_{*}$ is the pushforward map in rational cohomology. Until further notice we restrict to complex bundles and $K$-theory.

The simplest invariant of an element of $K(Z)$ after the rank, which is a continuous function $Z \rightarrow \mathbb{Z}$, is the determinant line bundle, which is a smooth complex line bundle over $Z$. In this topological context it is only defined up to equivalence.

A geometric family of Dirac operators parametrized by $Z$ is specified by a fiber bundle $\pi: X \rightarrow Z$, a spin structure on $X / Z$, a Riemannian metric on $X / Z$, and a distribution of horizontal planes on $X$ (transverse to the fibers $X / Z$ ). If we couple to a vector bundle $E \rightarrow X$, we require that $E$ have a metric and compatible connection. Then if the fibers $X / Z$ are closed, the determinant line bundle Det $D^{X / Z}(E)$ is welldefined as a smooth line bundle, and it carries a canonical metric and connection [BF]. If the fibers $X / Z$ are odd dimensional, so that the (complex) Dirac operator is selfadjoint, then there is a geometric invariant $\xi_{X / Z}(E): Z \rightarrow \mathbb{R} / \mathbb{Z}$ defined by AtiyahPatodi-Singer. (It is half the sum of the $\eta$-invariant and the dimension of the kernel.) Multiplying by $2 \pi \sqrt{-1}$ and exponentiating we obtain $\tau_{X / Z}(E): Z \rightarrow \mathbb{T}$, where $\mathbb{T} \subset \mathbb{C}$ is the unit circle.

The curvature of the determinant line bundle is the 2 -form

$$
\Omega^{\operatorname{Det} D^{X / Z}(E)}=\left[2 \pi \sqrt{-1} \int_{X / Z} \hat{A}\left(\Omega^{X / Z}\right) \operatorname{ch}\left(\Omega^{E}\right)\right]_{(2)} \in \Omega^{2}(Z),
$$

where $\Omega^{X / Z}, \Omega^{E}$ are the indicated curvature forms. As for the holonomy, consider a loop $\pi: X \rightarrow S^{1}$ of manifolds in this geometric setup. Endow $S^{1}$ with a metric and the bounding spin structure; then we induce a metric and spin structure on $X$. The holonomy of the determinant line bundle around this loop is

$$
\text { hol Det } D^{X / S^{1}}(E)=\operatorname{a-lim} \tau_{X}^{-1}(E)
$$

where the adiabatic limit a-lim is the limit as the metric on $S^{1}$ blows up $\left(g_{S^{1}} \rightarrow g_{S^{1}} / \epsilon^{2}\right.$ and $\epsilon \rightarrow 0$ ). If the determinant line bundle is flat, then no adiabatic limit is required. Equation (3.2) is the global anomaly formula [W1]; cf. [BF].

If $X$ is a spin manifold of odd dimension $n$, and $F \rightarrow X$ a flat unitary bundle of rank $r$, then for any complex hermitian bundle $E \rightarrow X$ with connection the ratio $\tau_{X}(E \otimes(F-r)) / \tau_{X}(E)$ is a topological invariant independent of the geometrical quantities. The flat index theorem of Atiyah-Patodi-Singer [APS2] gives a $K$-theory 
formula for this ratio. Namely, the bundle $F$ determines a class $[F-r] \in K^{-1}(Z ; \mathbb{R} / \mathbb{Z})$ and the difference of $\xi$-invariants is $\pi_{!}^{X}([E] \cdot[F-r])$, where now

$$
\pi_{!}^{X}: K^{-1}(Z ; \mathbb{R} / \mathbb{Z}) \rightarrow K^{-n-1}(\mathrm{pt} ; \mathbb{R} / \mathbb{Z}) \cong \mathbb{R} / \mathbb{Z} .
$$

If $X$ is an odd dimensional spin manifold with boundary, one can still define $\tau_{X}(E)$, but now it is an element of the inverse determinant line of the boundary, viewed as a $\mathbb{Z} / 2 \mathbb{Z}$-graded one dimensional vector space $[D F]$. Here we use the global boundary conditions of Atiyah-Patodi-Singer [APS1]. The invariant $\tau_{X}(E)$ satisfies a gluing law. We need the formula for $\tau_{-X}$, where $-X$ is the manifold $X$ with the opposite orientation. ${ }^{7}$ Let $k$ be the number of components of $\partial X$ on which the boundary Dirac operator has odd index. Then

$$
\tau_{-X}(E)=(-1)^{\left(\begin{array}{l}
k \\
2
\end{array}\right)} \tau_{X}(E)
$$

Let $X^{d}$ be the (spin) double of $X$, obtained by gluing $X$ and $-X$ along $\partial X$. Then the gluing theorem and (3.4) imply

$$
\tau_{X^{d}}=(-1)^{\left(\begin{array}{l}
k \\
2
\end{array}\right)}
$$

Turning now to real bundles and $K O$-theory, there is a square root which one can canonically extract in certain dimensions (see $[\mathrm{F} 1, \S 3]$ ). Namely, if a geometric family $\pi: X \rightarrow Z$ has $\operatorname{dim} X / Z \equiv 2(\bmod 8)$, and if $E \rightarrow X$ is a real vector bundle, then the determinant line bundle has a natural square root, the pfaffian line bundle Pfaff $D^{X / Z}(E)$. Also, if $X$ is a closed spin manifold with $\operatorname{dim} X \equiv 3(\bmod 8)$, and if $E \rightarrow X$ is a real vector bundle, then $\tau_{X}(E) \in \mathbb{T}$ has a natural square root $\tau_{X}^{1 / 2}(E) \in \mathbb{T}$. The curvature of the pfaffian line bundle is given by one-half times (3.1), and the holonomy by (3.2) with the $\tau^{-1 / 2}$-invariant replacing the $\tau^{-1}$-invariant. Formulas (3.4) and (3.5) hold for the $\tau^{-1 / 2}$-invariant, but now $k$ denotes the number of components of $\partial X$ with nonzero mod 2 index. (Note that the $\tau^{1 / 2}$-invariant of a manifold with boundary lives in the inverse pfaffian line of the boundary. That pfaffian line is $\mathbb{Z} / 2 \mathbb{Z}$-graded by the mod 2 index.) There is a version of the flat index theorem (3.3) which applies to this square root; it uses $K O$ in place of $K$.

There is a special low dimensional situation in which the topological isomorphism class of the pfaffian line bundle (over the integers) is computed by a cohomological formula. Suppose $\pi: X \rightarrow Z$ is a family of closed spin 2-manifolds, and $E \rightarrow X$ a virtual real vector bundle of rank 0 which is endowed with a spin structure. Then $[F 1, \S 5]$

$$
c_{1} \operatorname{Pfaff} D^{X / Z}(E)=\pi_{*}^{X / Z} \lambda(E),
$$

where $\lambda$ is the degree four characteristic class of spin bundles with $2 \lambda=p_{1}$. As a corollary, even if $E$ is not spin we have a cohomological formula for the determinant line bundle, which is the square of the pfaffian bundle:

$$
c_{1} \operatorname{Det} D^{X / Z}(E)=\pi_{*}^{X / Z} p_{1}(E) .
$$

In (5.22) we compute an analogous formula for the pfaffian line bundle of a family of Dirac operators on the circle.

\footnotetext{
${ }^{7}$ Unfortunately, the sign in (3.4) is missing from [DF]; it will be corrected in a forthcoming erratum.
} 


\section{Closed Superstrings.}

\section{Statement of Results}

In this section we study a closed superstring propagating in a curved background Riemannian manifold $Y$. Assume that $Y$ is an oriented manifold. Let $\Sigma$ be an oriented closed surface. We need not assume that either $Y$ or $\Sigma$ is connected. The space of bosons is the product

$$
B=\operatorname{Met}(\Sigma) \times \operatorname{Map}(\Sigma, Y)
$$

of Riemannian metrics $g$ on $\Sigma$ and maps $\phi: \Sigma \rightarrow Y$. A metric $g$ on $\Sigma$ induces a complex structure, since $\Sigma$ is assumed oriented, and so a $\bar{\partial}$ operator $\bar{\partial}_{g}: \Omega^{0,0} \rightarrow \Omega_{g}^{0,1}$. Let $K_{g}$ denote the canonical bundle, which is the cotangent bundle $T^{*} \Sigma$ viewed as a complex line bundle. A spin structure $\alpha$ on $\Sigma$, which we can describe on the oriented surface independent of any metric, gives rise to a complex line bundle $H_{\alpha, g}$ which satisfies $H_{\alpha, g}^{\otimes 2} \cong K_{g}$. It is natural to denote $H_{\alpha, g}$ as $K_{\alpha, g}^{1 / 2}$. Spinor fields are sections of $K_{\alpha, g}^{1 / 2} \oplus \overline{K_{\alpha, g}^{1 / 2}}$. The chiral Dirac operators on $\Sigma$ may be expressed in terms of the $\bar{\partial}$ operator and its conjugate:

$$
\begin{aligned}
& D_{\alpha, g}^{+}=\bar{\partial}_{g}: \Omega^{0,0}\left(K_{\alpha, g}^{1 / 2}\right) \longrightarrow \Omega_{g}^{0,1}\left(K_{\alpha, g}^{1 / 2}\right) \\
& \left.D_{\alpha, g}^{-}=-\partial_{g}: \Omega^{0,0} \overline{\left(K_{\alpha, g}^{1 / 2}\right.}\right) \longrightarrow \Omega_{g}^{0,1}\left(\overline{K_{\alpha, g}^{1 / 2}}\right)
\end{aligned}
$$

Note that $D^{-}=-\overline{D^{+}}$. Also, $D^{+}=-\left(D^{+}\right)^{*}$ is a complex skew-adjoint operator. For this we identify $\Omega^{0,1}\left(K^{1 / 2}\right)$ as the dual space to $\Omega^{0,0}\left(K^{1 / 2}\right)$ : the duality pairing is pointwise multiplication followed by integration. The determinant of a skew-adjoint operator has a canonical square root, the pfaffian, as explained in $\S 3$. For any vector bundle with connection $E \rightarrow \Sigma$ we can form the coupled Dirac operators $D^{ \pm}(E)$. The coupled operator is skew-adjoint if $E$ is real.

For a line bundle $\mathcal{L}$ we denote the dual by $\mathcal{L}^{-1}$ and the $n^{\text {th }}$ tensor power $\mathcal{L}^{\otimes n}$ by $\mathcal{L}^{n}$. Thus $K_{g}^{-1}$ is the holomorphic tangent bundle of $\Sigma$ and $K_{\alpha, g}^{-3 / 2}$ is the $3^{\text {rd }}$ power of the dual to $K_{\alpha, g}^{1 / 2}$.

Fix a spin structure $\alpha$. Then for a pair $(g, \phi) \in B$ define the complex line

$$
L_{\alpha}^{+}(g, \phi)=\operatorname{Pfaff} D_{\alpha, g}^{+}\left(\phi^{*} T Y\right) \otimes\left[\operatorname{Det} D_{\alpha, g}^{+}\left(K_{g}^{-1}\right)\right]^{\otimes(-1)} \otimes \operatorname{Det} \bar{\partial}_{g}\left(K_{g}^{-1}\right)
$$

and its complex conjugate

$$
L_{\alpha}^{-}(g, \phi)=\operatorname{Pfaff} D_{\alpha, g}^{-}\left(\phi^{*} T Y\right) \otimes\left[\operatorname{Det} D_{\alpha, g}^{-}\left(\bar{K}_{g}^{-1}\right)\right]^{\otimes(-1)} \otimes \operatorname{Det} \partial_{g}\left(\bar{K}_{g}^{-1}\right) .
$$

As $(g, \phi)$ vary these define smooth complex line bundles $L_{\alpha}^{ \pm} \rightarrow B$ with metric and connection. We remark that the last operator in (4.2) may be rewritten in terms of spinors:

$$
\bar{\partial}_{g}\left(K_{g}^{-1}\right)=D_{\alpha, g}^{+}\left(K_{\alpha, g}^{-3 / 2}\right)
$$

for any spin structure $\alpha$.

In superstring theory, the spin structures on right- and left-movers are chosen independently and are summed over with appropriate weighting. The path integral over the right-moving fermions is a section of $L_{\alpha}^{+}$, and the path integral over the leftmoving fermions is a section of $L_{\beta}^{-}$, for two independently chosen spin structures $\alpha$ and $\beta$. The first factor in the definition of $L_{\alpha}^{ \pm}$corresponds to the physical spinor field, the last factor to the ghosts from gauge fixing the diffeomorphism group, and the middle factor to the ghosts from gauge fixing the remainder of the superdiffeomorphism group. 
The path integral in the effective theory is carried out over the quotient of the space of bosons by the subgroup of superdiffeomorphisms of $\Sigma$ which preserve the chosen spin structure. Thus to detect possible anomalies we consider smooth fiber bundles $\pi: X \rightarrow Z$ with typical fiber $\Sigma$ together with a map to $Y$ :

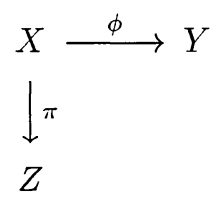

Furthermore, we assume that the relative tangent bundle carries a Riemannian metric and spin structure, and that there is a distribution of horizontal planes on $X$. This is enough to define the line bundles (4.2) and (4.3) with metric and connection. In the application to physics $Z$ maps into the quotient of $B$ by the subgroup of diffeomorphisms fixing the given spin structure. Such families of surfaces are "probes" which determine the structure of the line bundles $L_{\alpha}^{ \pm}$. It suffices to take $Z$ finite dimensional and compact. The path integral over both right- and left-moving fermions is a section of $L_{\alpha}^{+} \otimes L_{\beta}^{-}$, and it is the triviality of this line bundle over arbitrary families (4.4) which we investigate.

We now state the basic results for closed oriented surfaces; the proofs follow below. In these theorems isomorphism means an invertible linear map which preserves the metric and connection. A trivializable bundle is isomorphic to the trivial bundle with product metric and connection.

Theorem 4.5. Suppose $Y$ is spin and $\operatorname{dim} Y=10$. Then for any two spin structures $\alpha, \beta$ there exists an isomorphism

$$
L_{\alpha}^{+} \cong L_{\beta}^{+} \text {. }
$$

Since $L_{\alpha}^{-}$is the complex conjugate of $L_{\alpha}^{+}$, it follows that $L_{\alpha}^{-}$is also independent of the spin structure $\alpha$.

Corollary 4.6. Suppose $Y$ is spin and $\operatorname{dim} Y=10$. Then $L_{\alpha}^{+} \otimes L_{\beta}^{-}$is trivializable for any spin structures $\alpha, \beta$.

For $\alpha=\beta$ the hermitian metric provides the desired trivialization. The result for $\alpha \neq$ $\beta$ follows immediately from Theorem 4.5 . The triviality of $L_{\alpha}^{+} \otimes L_{\beta}^{-}$is the vanishing of the anomaly.

The next result is relevant to the conformal anomaly, but does not enter into the considerations of $\S 5$. We include it for completeness.

ThEOREM 4.7. Suppose $Y=\mathbb{R}^{10}$. Then $L_{\alpha}^{+} \otimes\left(\operatorname{Det} \bar{\partial}_{g}\right)^{\otimes(-5)}$ is trivializable.

The factor of $\left(\operatorname{Det} \bar{\partial}_{g}\right)^{\otimes(-5)}$ comes from integrating the boson $\phi$. (See [F2, $\left.\S 2\right]$ for a discussion of the conformal anomaly in the context of the bosonic string.)

Proofs

The proof ${ }^{8}$ of Theorem 4.5 is modelled on [FM], which treats a similar proposition for complex vector bundles. The real case treated here is simpler. First, the curvature formula (3.1) does not depend on the spin structure, so the ratio $L_{\alpha}^{+} / L_{\beta}^{+}$is flat. Thus

${ }^{8}$ For an alternative proof, see [W2]. 
we must show that the holonomy of this ratio vanishes. Now the holonomy of a pfaffian line bundle is given by the adiabatic limit of the exponentiated $-\xi / 2$-invariant of the Dirac operator on a 3-manifold which fibers over the circle (see (3.2)). Theorem 4.5 follows from a more general statement. ${ }^{9}$

LEMMA 4.8. Suppose $P$ is any compact oriented 3-manifold with two spin structures $\hat{\alpha}$ and $\hat{\beta}$, and $V \rightarrow P$ is a real vector bundle with rank divisible by 8 and with $w_{1}(V)=w_{2}(V)=0$. Then

$$
\frac{\xi_{\hat{\alpha}}}{2}-\frac{\xi_{\hat{\beta}}}{2} \equiv 0 \quad(\bmod 1)
$$

To prove Theorem 4.5 from this lemma, note first that the last factor in (4.2) does not depend on the spin structure. Next, use the fact that $D^{+}=\overline{D^{-}}$and $\operatorname{Det} D^{-}(E) \cong$ $\left(\operatorname{Det} D^{+}(E)\right)^{-1}$ for any complex vector bundle $E$ to rewrite the second factor in (4.2) as a pfaffian:

$$
\operatorname{Det} D_{\alpha, g}^{+}\left(K_{g}^{-1}\right) \cong \text { Pfaff } D_{\alpha, g}^{+}\left(K_{g}^{-1} \oplus{\overline{K_{g}}}^{-1}\right) .
$$

In this expression $K_{g}^{-1} \oplus{\overline{K_{g}}}^{-1}$ refers to a rank two real vector bundle. Now apply the lemma to $[V]=\phi^{*}[T Y]-\left[K_{g}^{-1} \oplus \bar{K}_{g}^{-1}\right]$. Assuming $Y^{10}$ to be oriented and spin, this has rank 8 and vanishing $w_{1}$ and $w_{2}$.

Proof of Lemma 4.8. A KO-theory version of the index theorem for flat bundles [APS2] gives a topological formula for this difference of $\xi / 2$-invariants (see (3.3)). Let $[V] \in K O(P)$ denote the $K O$-class of $V$. The difference of spin structures is a flat real bundle, and gives rise to a class $[\hat{\alpha}-\hat{\beta}] \in K O^{-1}\left(P ; \mathbb{Q} / \mathbb{Z}\right.$.). Let $\pi_{!}: K O^{-1}(P ; \mathbb{Q} / \mathbb{Z})$ $\rightarrow \mathbb{Q} / \mathbb{Z}$ be the direct image map defined by the spin structure $\hat{\beta}$. Then the flat index theorem asserts

$$
\frac{\xi_{\hat{\alpha}}}{2}-\frac{\xi_{\hat{\beta}}}{2} \equiv \pi_{!}([V] \cdot[\hat{\alpha}-\hat{\beta}]) \quad(\bmod 1) .
$$

In fact, we will show that

$$
[V] \cdot[\hat{\alpha}-\hat{\beta}]=0 \quad \text { in } K O^{-1}(P ; \mathbb{Q} / \mathbb{Z}) .
$$

First, an element $[V] \in K O(P)$ is determined by $\operatorname{rank}([V]), w_{1}([V])$, and $w_{2}([V])$. (An element of $K O(P)$ is a homotopy class of maps $P \rightarrow \mathbb{Z} \times B O$, and if the rank and first two Stiefel-Whitney classes are trivial the map lands in BSpin, which has trivial 3 -skeleton.) So with our hypotheses $[V]=8 k$ for some $k \in \mathbb{Z}$. Next, the difference of spin structures is given by a homotopy class of maps $P \rightarrow \mathbb{R}^{\infty}$, and since $P$ is 3 -dimensional by a map $P \rightarrow \mathbb{R P}^{4}$. The rational (reduced) $K O$ groups of $\mathbb{R} \mathbb{P}^{4}$ vanish, and the reduced group $\widetilde{K O}\left(\mathbb{R P}^{4}\right) \cong \mathbb{Z} / 8 \mathbb{Z}$, as was computed by Adams [A]. Hence $K O^{-1}\left(\mathbb{R} \mathbb{P}^{4} ; \mathbb{Q} / \mathbb{Z}\right) \cong \widehat{K O}\left(\mathbb{R P}^{4}\right) \cong \mathbb{Z} / 8 \mathbb{Z}$. It follows that $8[\hat{\alpha}-\hat{\beta}]=0$, whence (4.12).

Proof of Theorem 4.7. We must show that for any family $\pi: X \rightarrow Z$ of spin surfaces with the usual geometric data, the line bundle

$$
\mathcal{L}=\left(\operatorname{Det} D^{+}\right)^{5} \otimes(\operatorname{Det} \bar{\partial})^{-5} \otimes \operatorname{Det} \bar{\partial}\left(K^{-1}\right) \otimes\left(\operatorname{Det} D^{+}\left(K^{-1}\right)\right)^{-1}
$$

\footnotetext{
${ }^{9}$ It seems that there is no cohomological formula for this ratio of holonomies (i.e., the left hand side of (4.9)) if we relax the hypotheses $w_{1}(V)=w_{2}(V)=0$, as we learned in a conversation with John Morgan. Our proof of Lemma 4.8 is based on the $K O$-theory formula (4.11).
} 
over $Z$ is trivial. (For readability, we omit the metric $g$ and spin structure $\alpha$ from the notation in this proof.) That $\mathcal{L}$ is topologically trivial follows from a cohomological computation, thanks to (3.7). Namely, we can write $\mathcal{L}$ as the determinant line bundle of the Dirac operator $D^{+}$coupled to the virtual complex vector bundle

$$
[W]=\left[5-5 K^{-1 / 2}+K^{-3 / 2}-K^{-1}\right] \in K(X) .
$$

Let $x=c_{1}\left(K^{-1 / 2}\right) \in H^{2}(X)$. Then

$$
p_{1}([W])=-5 x^{2}+9 x^{2}-4 x^{2}=0,
$$

and so $c_{1}(\mathcal{L})=\pi_{*} p_{1}(W)=0$. The curvature of the natural connection on $\mathcal{L}$ also vanishes, since the curvature (3.1) is computed by a combination of differential forms with the same coefficients as in (4.13).

It remains to show that the holonomy is trivial. For this we rewrite $\mathcal{L}$ (canonically) as a product of pfaffian line bundles of Dirac operators coupled to real vector bundles. (For the last factor, see (4.10)):

$$
\begin{aligned}
\mathcal{L}=\left(\text { Pfaff } D^{+}\right)^{10} & \otimes\left(\text { Pfaff } D^{+}\left(K^{-1 / 2} \oplus \bar{K}^{-1 / 2}\right)\right)^{-5} \\
& \otimes\left(\text { Pfaff } D^{+}\left(K^{-3 / 2} \oplus \bar{K}^{-3 / 2}\right)\right) \otimes\left(\text { Pfaff } D^{+}\left(K^{-1} \oplus \bar{K}^{-1}\right)\right)^{-1} .
\end{aligned}
$$

Consider a family of surfaces $P \rightarrow S^{1}$ fibered over the circle. The holonomy of $\mathcal{L}$ around $S^{1}$ is the adiabatic limit of the product of $\tau^{-1 / 2}$-invariants of the Dirac operator $D_{P}$ on $P$ coupled to the real vector bundles indicated in (4.14). These bundles are associated to the relative tangent bundle of the fibering $P \rightarrow S^{1}$. We rewrite these coupled Dirac operators as operators which make sense on any spin 3-manifold. (Below we write $P=\partial W$ for a spin 4-manifold $W$ and we want to extend the operators over $W$, which may not fiber over the circle.) For example, since $T P \otimes \mathbb{C} \cong K^{-1} \oplus \bar{K}^{-1} \oplus 1_{\mathbb{C}}$, the operators which appear in the first and last factors in (4.14) may be combined as

$$
D_{P}(11-T P) \text {, }
$$

where 11 is the trivial real bundle of rank 11. (What we really compute is the $11^{\text {th }}$ power of the $\tau^{-1 / 2}$-invariant of $D_{P}$ divided by the $\tau^{-1 / 2}$-invariant of $D_{P}(T P)$, but it is convenient to use virtual bundles as a shorthand for this.) For the second factor in (4.14) we rewrite $D_{P}\left(K^{-1 / 2} \oplus \bar{K}^{-1 / 2}\right)$ on the fibered manifold $P \rightarrow S^{1}$ as the operator ${ }^{10}$

$$
B^{\mathrm{ev}}=(-1)^{p}(* d-d *)
$$

acting on $\Omega^{0}(P) \oplus \Omega^{2}(P)$, where $p=0$ on $\Omega^{0}$ and $p=1$ on $\Omega^{2}$. This operator is "half" the boundary of the four dimensional signature operator, as explained in [APS1]. An important point for us is that its $\xi$-invariant is well-defined as a real number, since the kernel of $B^{\text {ev }}$ has a cohomological interpretation so has constant dimension in families. Hence $\xi / 2(\bmod 1)$ is well-defined. For the third term of $(4.14)$ we rewrite $D_{P}\left(K^{-3 / 2} \oplus \bar{K}^{-3 / 2}\right)$ on the fibered manifold as

$$
B^{\mathrm{ev}}(T P)-2 B^{\mathrm{ev}} \text {. }
$$

Thus the operators which appear in the second and third factors in (4.14) may be combined as

$$
B^{\mathrm{ev}}(T P-7)
$$

${ }^{10}$ The notation " $B^{\mathrm{ev}}$ " is taken from [APS1]. 
Now we use the fact that any spin 3-manifold $P$ bounds a spin 4-manifold $W$. Then by the Atiyah-Patodi-Singer index theorem for manifolds with boundary [APS1], the $\tau^{-1 / 2}$-invariant is the exponential of a curvature integral over $W$. The particular curvature polynomial is determined from (4.15) and (4.16) to be the degree four part of a form we denote schematically by

$$
-\frac{1}{2} \hat{A}(W)(12-\operatorname{ch}(T W))-\frac{1}{4} L(W)(\operatorname{ch}(T W)-8) .
$$

Note that $\left.T W\right|_{P} \cong T P \oplus 1$, which explains the "12" and "8" in (4.17). Also, $L(W)$ is the unstable characteristic class based on the formal expression $\frac{x}{\tanh x / 2}$, as explained in [AS1,p. 577]. Expressed in terms of the first Pontrjagin polynomial $p_{1}$ in the curvature, the degree four part of (4.17) is the coefficient of $p_{1}$ in

$$
-\frac{1}{2}\left(1-\frac{p_{1}}{24}\right)\left(8-p_{1}\right)-\frac{1}{4}\left(4+\frac{p_{1}}{3}\right)\left(p_{1}-4\right),
$$

which vanishes. Thus the holonomy of $\mathcal{L}$ is trivial.

\section{Open Superstrings and D-Branes.}

\section{Statement of Results}

Fix a Riemannian manifold $Y$, which we assume to be oriented, spin, and of dimension 10. As before, this is the background in which the string propagates; now we want to add a D-brane. Thus let $Q \subset Y$ be an oriented submanifold, which we need not assume to be connected. The oriented (not necessarily connected) surface $\Sigma$ is now permitted to have a (not necessarily connected) boundary, and we require the boundary of $\Sigma$ to map to $Q$. In other words, the space of bosons is

$$
B=\operatorname{Met}(\Sigma) \times \operatorname{Map}((\Sigma, \partial \Sigma),(Y, Q)) .
$$

Asking that $\partial \Sigma$ map to $Q$ imposes a mixture of Dirichlet and Neumann boundary conditions. To define the determinant lines (4.2) and (4.3), we need to impose boundary conditions on the fermions; the desired boundary conditions are local boundary conditions that are determined by supersymmetry. These boundary conditions mix right- and left-handed spinor fields, so it is only the tensor product of the lines $\mathcal{L}^{+}$ and $\mathcal{L}^{-}$which makes sense if the boundary is nonempty.

To describe precisely the desired Dirac operators on $\Sigma$, we first recall that a spin structure $\alpha$ on $\Sigma$ induces a spin structure on $\partial \Sigma$. To see this, fix a metric $g$ on $\Sigma$ and consider the principal $S_{2}$-bundle of oriented orthonormal frames $S O(\Sigma) \rightarrow \Sigma$. Its restriction to $\partial \Sigma$ is canonically trivialized by the oriented orthonormal frame whose first element is the outward pointing unit normal. A spin structure $\alpha$ induces a double cover $\operatorname{Spin}(\Sigma) \rightarrow S O(\Sigma)$, and the inverse image of the trivialization at the boundary is a spin structure on the boundary. That inverse image is a double cover of $\partial \Sigma$. There are two possibilities on each component of $\partial \Sigma$. If the double cover is connected, then we say that the spin structure on that component is trivial, since it bounds a spin structure on a disk. If the double cover is not connected, then we say that the spin structure on that component is nontrivial. The spin structure on the entire boundary $\partial \Sigma$ is constrained by the fact that it is the boundary of a spin structure on $\Sigma$. As for the complex line bundle $K_{\alpha, g}^{1 / 2}$, it follows from this discussion that its restriction to $\partial \Sigma$ has a canonical real structure, i.e.,

$$
K_{\alpha, g}^{1 / 2} \cong \overline{K_{\alpha, g}^{1 / 2}} \quad \text { on } \partial \Sigma \text {. }
$$


The underlying real bundle determines the spin structure on $\partial \Sigma$.

As for closed strings we allow different spin structures on the right- and leftmovers. Thus we consider pairs of spin structures $\alpha, \beta$ such that the induced spin structures on $\partial \Sigma$ are isomorphic. Now an isomorphism of spin structures on $\partial \Sigma$ is only determined up to a sign on each component of $\partial \Sigma$, and we need to fix that sign. (The overall sign is irrelevant.) Thus part of the data we need is an isomorphism

$$
\theta:\left.\left.K_{\alpha}^{1 / 2}\right|_{\partial \Sigma} \longrightarrow K_{\beta}^{1 / 2}\right|_{\partial \Sigma}
$$

Once the isomorphism is chosen for some metric it is determined for all metrics, so (5.2) is a discrete topological choice. Thus the topological data is a triple $(\alpha, \beta, \theta)$.

Let $D$ denote the total Dirac operator, which is the sum of the two chiral operators (4.1). For a map $\phi: \Sigma \rightarrow Y$ with $\phi(\partial \Sigma) \subset Q$ and a metric $g$ on $\Sigma$, consider the total Dirac operator $D_{\alpha, \beta, \theta, g}\left(\phi^{*} T Y\right)$, where we use the spin structure $\alpha$ on right-handed spinors and the spin structure $\beta$ on left-handed spinor fields. The isomorphism $\theta$ does not enter into the definition of the Dirac operator, but is included in the notation since it does enter into the boundary condition (5.3) below. A spinor field $\psi$ with values in $\phi^{*} T Y$ decomposes as $\psi=\psi^{+}+\psi^{-}$according to the chiral decomposition of spinors on $\Sigma$. The restriction $\partial \psi$ of $\psi$ to $\partial \Sigma$ takes values in $\phi^{*}\left(\left.T Y\right|_{Q}\right)$. Now

$$
\left.T Y\right|_{Q} \cong T Q \oplus \nu
$$

where $\nu$ is the normal bundle to $Q$ in $Y$. Thus at the boundary we can write

$$
\partial \psi^{ \pm}=\lambda_{Q}^{ \pm}+\lambda_{\nu}^{ \pm}
$$

The boundary condition for the operator $D_{\alpha, \beta, \theta, g}\left(\phi^{*} T Y\right)$ is then

$$
\begin{array}{ll}
D_{\alpha, \beta, \theta, g}\left(\phi^{*} T Y\right): & \theta\left(\lambda_{Q}^{+}\right)=\lambda_{Q}^{-} \\
& \theta\left(\lambda_{\nu}^{+}\right)=-\lambda_{\nu}^{-}
\end{array}
$$

The operator $D_{\alpha, \beta, \theta, g}\left(\phi^{*} T Y\right)$ with these boundary conditions is complex skew-adjoint.

There are two other operators - acting on the ghost fields-which enter. They appear in the determinant line of interest:

$$
\begin{aligned}
L_{\alpha, \beta, \theta}(g, \phi)=\text { Pfaff } D_{\alpha, \beta, \theta, g}\left(\phi^{*} T Y\right) \otimes\left[\operatorname{Det} D_{\alpha, \beta, \theta, g}\left(K_{g}^{-1} \oplus \overline{K_{g}}-1\right)\right]^{-1} & \\
& \otimes \operatorname{Det}\left(\bar{\partial}_{g}\left(K_{g}^{-1}\right) \oplus \partial_{g}\left(\bar{K}_{g}-1\right)\right) .
\end{aligned}
$$

For each of the last two factors we ask that the two fields which appear be equal on $\partial \Sigma$. This boundary condition makes sense, since on $\partial \Sigma$ there is a natural real structure on the inverse canonical bundle $K_{g}^{-1}$. Note that in the second factor the boundary condition involves the isomorphism $\theta$, as in (5.3). The field in the third factor is a complexified tangent vector to the surface, and the boundary condition is the complexification of the condition that a real tangent vector be tangent to the boundary. Thus the domain of the operator is the complexification of the group of infinitesimal diffeomorphisms. The boundary condition in the second factor is the odd analog for superdiffeomorphisms.

We study $L_{\alpha, \beta, \theta}$ as a line bundle over $Z$ for families (4.4), where now $X$ has a 
boundary which fibers over $Z$ with typical fiber $\partial \Sigma$ and $\phi(\partial X) \subset Q$ :

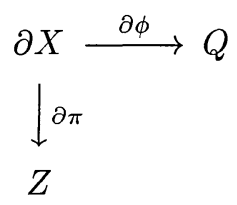

Our first result computes the topology — that is, the first Chern class-of the line bundle $L_{\alpha, \beta, \theta}$.

THEOREM 5.5. The isomorphism class of the complex line bundle $L_{\alpha, \beta, \theta}$ equals $(\partial \pi)_{*}(\partial \phi)^{*} W_{3}(\nu)$ in $H^{2}(Z ; \mathbb{Z})$, where $W_{3}$ is the third Stiefel-Whitney class. In fact, this line bundle is the complexification of a real line bundle, and the isomorphism class in $H^{1}(Z ; \mathbb{Z} / 2 \mathbb{Z})$ of the underlying real line bundle equals $(\partial \pi)_{*}(\partial \phi)^{*} w_{2}(\nu)$.

Here $(\partial \pi)_{*}$ denotes integration along the fibers of $\partial \pi$. The first statement follows from the second by applying the Bockstein homomorphism, which commutes with pushforward and pullback.

As explained at the beginning of $\S 3$, for the application to anomalies the topological isomorphism class is not fine enough. One needs also to compute the isomorphism class of the canonical connection-its curvature and holonomy. The theory of the Quillen metric and canonical connection on the determinant line bundle only exists in the literature for families of closed manifolds [BF], or families of manifolds with boundary and global boundary conditions of Atiyah-Patodi-Singer type [P]. In our problem we have a family of manifolds with boundary and local boundary conditions. As we will see below, by gluing we identify the index problem on surfaces with boundary with an index problem on the double manifold, and then we can apply the usual geometric theory of determinant line bundles on closed manifolds. In $\S 6$ we describe a general class of Dirac operators with local boundary conditions and the associated doubling.

Let $X^{d} \rightarrow Z$ be the family of doubled surfaces, and $\gamma=\gamma(\alpha, \beta, \theta)$ the spin structure on the double obtained by gluing $\alpha, \beta$ using $\theta$. As part of the proof of Theorem 5.5 we identify $L_{\alpha, \beta, \theta}$ with a twisted version $\tilde{L}_{\gamma}^{+}$of (4.2). Of course, the topology of $\tilde{L}_{\gamma}^{+}$is determined by $W_{3}(\nu)$, as in Theorem 5.5, but our main result asserts that the holonomy of its natural connection is given by the second Stiefel-Whitney class $w_{2}(\nu)$.

THEOREM 5.6. The canonical connection on $\tilde{L}_{\gamma}^{+}$is flat. Consider a family of surfaces with boundary $\pi: X \rightarrow S^{1}$ and the associated family of doubles $\pi^{d}: X^{d} \rightarrow S^{1}$. Then the holonomy of the canonical connection around this loop is \pm 1 with the sign given by $(\partial \phi)^{*} w_{2}(\nu)[\partial X]$. (See (5.4) for the notation.)

\section{Proofs}

The proof of Theorem 5.5 proceeds in two main steps. Define an operator $D_{\alpha, \beta, \theta, g}^{\prime}\left(\phi^{*} T Y\right)$ which differs from $D_{\alpha, \beta, \theta, g}\left(\phi^{*} T Y\right)$ only by a sign in the boundary condition:

$$
\begin{array}{ll}
D_{\alpha, \beta, \theta, g}^{\prime}\left(\phi^{*} T Y\right): & \theta\left(\lambda_{Q}^{+}\right)=\lambda_{Q}^{-} \\
\theta\left(\lambda_{\nu}^{+}\right)=\lambda_{\nu}^{-}
\end{array}
$$


The operator $D_{\alpha, \beta, \theta, g}^{\prime}\left(\phi^{*} T Y\right)$ is also complex skew adjoint. Then set

$$
\begin{aligned}
L_{\alpha, \beta, \theta}^{\prime}(g, \phi)=\operatorname{Pfaff} D_{\alpha, \beta, \theta, g}^{\prime}\left(\phi^{*} T Y\right) \otimes\left[\operatorname{Det} D_{\alpha, \beta, \theta, g}\left(K_{g}^{-1} \oplus \overline{K_{g}}-1\right)\right]^{-1} & \\
& \otimes \operatorname{Det}\left(\bar{\partial}_{g}\left(K_{g}^{-1}\right) \oplus \partial_{g}\left(\bar{K}_{g}-1\right)\right) .
\end{aligned}
$$

We first show the following.

Proposition 5.8. The ratio $L_{\alpha, \beta, \theta} \otimes\left(L_{\alpha, \beta, \theta}^{\prime}\right)^{-1}$ is the complexification of a real line bundle whose isomorphism class in $H^{1}(Z ; \mathbb{Z} / 2 \mathbb{Z})$ is $(\partial \pi)_{*}(\partial \phi)^{*} w_{2}(\nu)$.

In the process of proving Proposition 5.8 we identify $L_{\alpha, \beta, \theta}^{\prime}$ with the line bundle $L_{\gamma}^{+}$(see (4.2)) on the double $X^{d}$, where as above $\gamma=\gamma(\alpha, \beta, \theta)$ is the spin structure on the double obtained by gluing $\alpha, \beta$ using $\theta$. We also identify $L_{\alpha, \beta, \theta}$ with a twisted version $\tilde{L}_{\gamma}^{+}$. The first step towards proving Theorem 5.6 is a geometric version of Proposition 5.8.

Proposition 5.9. The canonical connection on the ratio $\tilde{L}_{\gamma}^{+} \otimes\left(L_{\gamma}^{+}\right)^{-1}$ is flat, and the holonomy for any family of closed surfaces $\pi: \mathcal{X}^{d} \rightarrow S^{1}$ is \pm 1 with the sign given by $(\partial \phi)^{*} w_{2}(\nu)[\partial \mathcal{X}]$.

The second step in the proofs of Theorem 5.5 and Theorem 5.6 is the following.

Proposition 5.10. $L_{\gamma}^{+}$is trivializable (as a bundle with metric and connection) over a family of doubled surfaces.

We prove Proposition 5.10 directly only for the symmetric case $\alpha=\beta$; the result for arbitrary $\alpha, \beta$ then follows from Theorem 4.5 .

We give three proofs of Proposition 5.8. Notice that the line bundle in question is

$$
\mathcal{L}_{\alpha, \beta, \theta}:=\left(\text { Pfaff } D_{\alpha, \beta, \theta}\right) \otimes\left(\text { Pfaff } D_{\alpha, \beta, \theta}^{\prime}\right)^{-1}
$$

First Proof of Proposition 5.8. A general result [BW, Theorem 21.2] for elliptic boundary-value problems (the "Agranovič-Dynin formula") describes the dependence of the index on local boundary conditions. The version we need is for families of complex skew-adjoint Dirac operators, and it follows from [N,Theorem 6.2]. It computes the difference of the $K O$-indices of $D_{\alpha, \beta, \theta}$ and $D_{\alpha, \beta, \theta}^{\prime}$ as the index of a family of operators on the boundary family (5.4):

$$
\operatorname{ind}^{X / Z}\left(D_{\alpha, \beta, \theta}\right)-\operatorname{ind}^{X / Z}\left(D_{\alpha, \beta, \theta}^{\prime}\right)=\operatorname{ind}^{\partial X / Z}\left(R P R^{\prime *}\right) \quad \in K O^{-2}(Z) .
$$

We must explain the operators $P, R, R^{\prime}$ on the right hand side.

First, $P$ is a zeroth order pseudodifferential operator, the Calderón projector. We only need its principal symbol. For that, recall that the spinor fields on the boundary are sections of

$$
\left(K_{\alpha}^{1 / 2} \oplus \bar{K}_{\beta}^{1 / 2}\right) \otimes(\partial \phi)^{*}(T Q \oplus \nu) .
$$

Also, using (5.1) and (5.2) we identify the two spinor bundles as a single real line bundle $S$. For simplicity introduce the notation

$$
\begin{aligned}
F & :=(\partial \phi)^{*} T Q \\
F^{\prime} & :=(\partial \phi)^{*} \nu
\end{aligned}
$$


Then we rewrite (5.13) as the real bundle

$$
(S \oplus S) \otimes\left(F \oplus F^{\prime}\right) \cong\left(S \otimes\left(F \oplus F^{\prime}\right)\right) \oplus\left(S \otimes\left(F \oplus F^{\prime}\right)\right)
$$

Let $\not D$ be the standard Dirac operator on the boundary circle. Then the operator which acts on (5.14) - the "boundary operator" associated to $D_{\alpha, \beta, \theta}$ (see [BW,Theorem $12.4])$ - is $\left(\begin{array}{ll}-\not D\end{array}\right)$ relative to the decomposition (5.15). The principal symbol of this operator is invertible and self-adjoint. The Caldéron projector $P$ has a principal symbol $\sigma(P)$ which is the projection onto the sum of the eigenspaces with positive eigenvalues. Thus for a nonzero cotangent vector $\xi$ on the boundary,

$$
\sigma(P)(\xi)= \begin{cases}\left(\begin{array}{ll}
1 & \\
& 0
\end{array}\right), & \xi>0 \\
\left(\begin{array}{ll}
0 & \\
& 1
\end{array}\right), & \xi<0\end{cases}
$$

where we use the orientation of the boundary to give meaning to the sign of $\xi$.

The operators $R, R^{\prime}$ in (5.12) are vector bundle maps

$$
R, R^{\prime}:\left(S \otimes\left(F \oplus F^{\prime}\right)\right) \oplus\left(S \otimes\left(F \oplus F^{\prime}\right)\right) \longrightarrow S \otimes\left(F \oplus F^{\prime}\right)
$$

whose kernel consists of spinor fields which satisfy the local boundary conditions (5.3), (5.7). Relative to the decompositions shown in (5.17), we write these operators as the matrices

$$
\begin{aligned}
R & =\left(\begin{array}{ll}
1 \oplus 1 & -1 \oplus 1
\end{array}\right), \\
R^{\prime} & =\left(\begin{array}{ll}
1 & -1
\end{array}\right) .
\end{aligned}
$$

Since $R, R^{\prime}$ are vector bundle maps, they are equal to their principal symbols.

Now the principal symbol of the operator $R P R^{\prime *}$ which appears in (5.12) is easily computed from (5.16) and (5.18):

$$
\sigma\left(R P R^{\prime *}\right)(\xi)= \begin{cases}\left(\begin{array}{ll}
1 \oplus 1 & -1 \oplus 1
\end{array}\right)\left(\begin{array}{ll}
1 & \\
& 0
\end{array}\right)\left(\begin{array}{r}
1 \\
-1
\end{array}\right)=(1), & \xi>0 \\
\left(\begin{array}{ll}
1 \oplus 1 & -1 \oplus 1
\end{array}\right)\left(\begin{array}{l}
0 \\
\\
\end{array}\right)\left(\begin{array}{r}
1 \\
-1
\end{array}\right)=(1 \oplus-1), & \xi<0 .\end{cases}
$$

These matrices act on $S \otimes\left(F \oplus F^{\prime}\right)$. Observe that (5.19) is also the symbol of the family of operators

$$
\operatorname{id}_{S \otimes F} \oplus \not D\left(F^{\prime}\right)
$$

on $\partial X \rightarrow Z$. Since the index only depends on the symbol, and since the index of the identity operator is trivial, we see that (5.12) reduces to

$$
\operatorname{ind}^{X / Z}\left(D_{\alpha, \beta, \theta}\right)-\text { ind }^{X / Z}\left(D_{\alpha, \beta, \theta}^{\prime}\right)=\text { ind }^{\partial X / Z}\left(\not D\left(F^{\prime}\right)\right) .
$$

The index we are computing lies in $K \mathrm{O}^{-2}(Z)$; it is the index of a family of complex skew-adjoint operators. But $\not D\left(F^{\prime}\right)$ is a family of real skew-adjoint operators, so its index lies naturally in $K O^{-1}(Z)$. The index we seek in $K O^{-2}(Z)$ is thus the image of ind $^{\partial X / Z} \not D\left(F^{\prime}\right)$ under the natural map

$$
K O^{-1}(Z) \stackrel{[\eta-1]}{\longrightarrow} K O^{-1}(\mathrm{pt}) \otimes K O^{-1}(Z) \cong K O^{-2}(Z),
$$

where $\eta \in K O\left(S^{1}\right)$ is the Möbius bundle. We are interested in the complex pfaffian line bundle, which in this situation is the complexification of the real pfaffian line 
bundle:

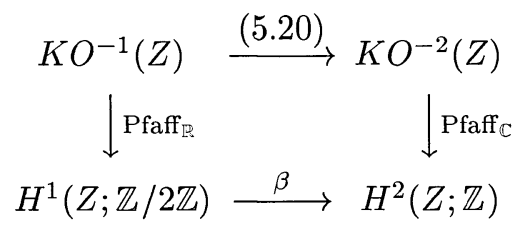

Here $\beta$ is the Bockstein map. So we are reduced to demonstrating a well-known result: for any fibering $\rho: W \rightarrow Z$ of spin 1-manifolds and any oriented real vector bundle $F^{\prime} \rightarrow W$

$$
\operatorname{Pfaff}_{\mathbb{R}} \not D^{W / Z}\left(F^{\prime}\right)=\rho_{*} w_{2}\left(F^{\prime}\right) .
$$

To check (5.22) it suffices to take $Z=S^{1}$, so $W=S^{1} \times S^{1}$. Endow $Z$ with the bounding spin structure; then the left hand side of (5.22), evaluated on the fundamental class of $Z$, is the image of ind ${ }^{W / Z} \not D\left(F^{\prime}\right)$ under

$$
\pi_{!}^{Z}: K O^{-1}\left(S^{1}\right) \longrightarrow K O^{-2}(\mathrm{pt}) \cong \mathbb{Z} / 2 \mathbb{Z} .
$$

By the multiplicative property of the direct image--essentially the Thom isomorphism in $K O$-theory - this is $\pi_{!}^{W}\left(\left[F^{\prime}\right]\right)$ for

$$
\pi_{!}^{W}: K O(W) \longrightarrow K O^{-2}(\mathrm{pt}) \cong \mathbb{Z} / 2 \mathbb{Z}
$$

the direct image map. (By the index theorem [AS2] this is the mod 2 index of the Dirac operator on $W$ coupled to the real vector bundle $F^{\prime}$.) Since $W$ bounds a spin manifold, we have $\pi_{!}^{W}([1])=0$, and so

$$
\pi_{!}^{W}\left(\left[F^{\prime}\right]\right)=\pi_{!}^{W}\left(\left[F^{\prime}-\operatorname{dim} F^{\prime}\right]\right) .
$$

Now $x=\left[F^{\prime}-\operatorname{dim} F^{\prime}\right] \in \widetilde{K O}(W)$ satisfies $w_{1}(x)=w_{1}\left(F^{\prime}\right)=0$, so is determined by $w_{2}(x)=w_{2}\left(F^{\prime}\right)$. We can arrange the support of $x$ to be contained in a disk in $W$, and by excision it follows that $\pi_{!}^{W}(x)=\pi_{!}^{S^{2}}(y)$ for $y \in \widetilde{K O}\left(S^{2}\right)$ with $w_{2}(y)\left[S^{2}\right]=$ $w_{2}(x)[W]$. Finally, the direct image map

$$
\pi_{!}^{S^{2}}: \widetilde{K O}\left(S^{2}\right) \longrightarrow K O^{-2}(\mathrm{pt}) \cong \mathbb{Z} / 2 \mathbb{Z}
$$

is an isomorphism, and the generator of $\widetilde{K O}\left(S^{2}\right)$ is a bundle with $w_{2} \neq 0$.

For the other proofs of Proposition 5.8 we introduce the fibered double $X^{d} \rightarrow Z$, which is a family of closed oriented surfaces obtained by gluing $X \cup-X$ along the boundary $\partial X$. Also, the spin structure $\alpha$ on $X / Z$ and $\beta$ on $-X / Z$ glue to a spin structure $\gamma$ on $X^{d} / Z$ via the isomorphism $\theta$ in (5.2). A right-handed spinor field on $X_{\gamma}^{d} / Z$ can be identified with a pair $\left(\psi^{+}, \psi^{-}\right): \psi^{+}$is a right-handed spinor field on $X_{\alpha}, \psi^{-}$is a left-handed spinor field on $X_{\beta}$, and $\psi^{+}=\psi^{-}$on $\partial X$ using $\theta$. So the operator $D_{\alpha, \beta, \theta, g}^{\prime}\left(\phi^{*} T Y\right)$ (see (5.7)) may be identified ${ }^{11}$ with the chiral Dirac operator $D_{\gamma, g}^{+}\left(\phi^{*} T Y\right)$ on the double. Note that $X^{d} / Z$ has an orientation-reversing involution, but it does not lift to the spin bundle unless $\alpha=\beta$ and $\theta$ is the identity.

Next, we rewrite the twisted boundary conditions (5.3) on the double, for simplicity on a single surface $\Sigma$. Then in the double $\Sigma^{d}$ there is a collar neighborhood

11 The identification of the indices under gluing is discussed in a similar situation in [F3, $\S 2]$. The gluing indicated here only gives continuous spinor fields on the double; it is nicer to glue along an open cylinder near the boundary, as in [DF,§IV]. We generalize and give more details in the second part of section 6 . 
$I \times \partial \Sigma \subset \Sigma^{d}$ of $\partial \Sigma$, where $I \simeq(-1,1)$. Over that neighborhood we have a splitting $\phi^{*} T Y \cong F \oplus F^{\prime}$, using the notation of (5.14). Let $\eta$ be the real line bundle on $\Sigma^{d}$ obtained by identifying the trivial real line bundle on $\Sigma$ with the trivial real line bundle on $-\Sigma$ via the isomorphism -1 on the collar region. Then $\eta$ is canonically trivial away from the collar, and there is a real vector bundle $E \rightarrow \Sigma^{d}$ which is canonically $\phi^{*} T Y$ away from the collar and $F \oplus\left(\eta \otimes F^{\prime}\right)$ on the collar. We identify $D_{\alpha, \beta, \theta, g}\left(\phi^{*} T Y\right)$ on $\Sigma$ with $D_{\gamma, g}^{+}(E)$ on $\Sigma^{d}$; the line bundle $\eta$ incorporates the sign in (5.3). Therefore, the ratio (5.11) for a family $X \rightarrow Z$ of surfaces with boundary is

$$
\operatorname{Pfaff}_{\mathbb{C}} D_{\gamma}^{+}\left((\eta-1) \otimes F^{\prime}\right)
$$

for the family of chiral Dirac operators on the relative double $X^{d} \rightarrow Z$. Note that $\eta-1$ is supported in the collar region $I \times \partial X$ and is pulled back from the first factor. We identify

$$
[\eta-1] \in K O(I, \partial I)
$$

as the (Hopf bundle) generator. Also, up to isomorphism $F^{\prime}$ is pulled back from the second factor $\partial X$ :

$$
\left[F^{\prime}\right]=\left[(\partial \phi)^{*} \nu\right] \in K O(\partial X) .
$$

Second proof of Proposition 5.8. By the excision property of the index, (5.25) is

$$
\operatorname{Pfaff}_{\mathbb{C}} \pi_{!}^{(I, \partial I) \times \partial X / Z}\left((\eta-1) \otimes\left[F^{\prime}\right]\right)
$$

where

$$
\pi_{!}^{(I, \partial I) \times \partial X / Z}: K O((I, \partial I) \times \partial X) \longrightarrow K O^{-2}(Z)
$$

is the direct image map. This factors as

$\pi_{!}^{(I, \partial I) \times \partial X / Z}\left((\eta-1) \otimes\left[F^{\prime}\right]\right)=\pi_{!}^{(I, \partial I)}([\eta-1]) \cdot \pi_{!}^{\partial X / Z}\left(\left[F^{\prime}\right]\right) \in K O^{-1}(\mathrm{pt}) \otimes K O^{-1}(Z)$.

Multiplication by $[\eta-1]$ is $(5.20)$, and the computation of $\pi_{!}^{\partial X / Z}\left(\left[F^{\prime}\right]\right)$ proceeds as in the first proof. (See (5.22) and the argument which follows.)

Third proof of Proposition 5.8. Assume first that $\operatorname{rank} F^{\prime}$ is even. Write $x=$ $(\eta-1) \otimes F^{\prime}$. Then from the Whitney sum formula we compute the Stiefel-Whitney classes of the virtual bundle $x$ :

$$
\begin{aligned}
& w_{1}(x)=w_{2}(x)=w_{3}(x)=0, \\
& w_{4}(x)=w_{1}(\eta) \smile w_{3}\left(F^{\prime}\right) .
\end{aligned}
$$

Thus $x$ is orientable and spinable, so by (3.6) the isomorphism class of the pfaffian line bundle is a pushforward in cohomology:

$$
c_{1} \text { Pfaff } D_{\gamma}^{+}(x)=\pi_{*}^{X^{d} / Z}(\lambda(x)),
$$

where

$$
\pi_{*}^{X^{d} / Z}: H^{4}\left(X^{d}\right) \longrightarrow H^{2}(Z)
$$

and $\lambda$ is the characteristic class of a spin bundle with $2 \lambda=p_{1}$. By excision, we compute on the tubular neighborhood $(I, \partial I) \times \partial X$ and extend to $X^{d}$. We claim

$$
\lambda(x)=\beta\left(w_{1}(\eta) \smile w_{2}\left(F^{\prime}\right)\right) \in H^{4}((I, \partial I) \times \partial X) ;
$$

then Proposition 5.8 follows from (5.21) and the fact that the Bockstein $\beta$ commutes with pushforward. To prove the claim, we work in the universal situation where the 
bundle $x=(\eta-1) \otimes F^{\prime}$ lives over $S^{1} \times B S O$. First, since $\eta \oplus \eta$ is trivial, it follows that $\lambda(x)$ is torsion of order two. Since all of the torsion in $H^{\bullet}(B S O)$ has order two (see $[\mathrm{BH}, \S 30]$ ) it follows that $\lambda(x)$ is determined by its image in real cohomology, which vanishes, and its image in $\mathbb{Z} / 2 \mathbb{Z}$ cohomology, which is $w_{4}(x)=w_{1}(\eta) \smile w_{3}\left(F^{\prime}\right)$. The integral class in (5.27) is also torsion of order two and reduces $\bmod 2$ to $w_{1}(\eta) \smile$ $w_{3}\left(F^{\prime}\right)$.

If rank $F^{\prime}$ is odd, we replace $F^{\prime}$ with $F^{\prime}$ plus a trivial line bundle and apply the previous. So we are reduced to proving that Pfaff $D_{\gamma}^{+}(\eta-1)$ is trivial. This follows from excision and the factorization (5.26).

We now prove Proposition 5.9. Notice that the ratio of bundles which appears in this proposition is $(5.25)$.

Proof of Proposition 5.9. The curvature (3.1) vanishes since virtual bundle $(\eta-$ $1) \otimes F^{\prime}$ has rank zero and is flat. To compute the holonomy (3.2) around a family of closed manifolds $\pi: \mathcal{X} \rightarrow S^{1}$, we endow the base $S^{1}$ with the bounding spin structure and induce a spin structure on $\mathcal{X}$; then the holonomy is the ratio of the $\tau^{-1 / 2}$-invariant of $\eta \otimes F^{\prime} \rightarrow \mathcal{X}$ to the $\tau^{-1 / 2}$-invariant of $F^{\prime} \rightarrow \mathcal{X}$. (There is no adiabatic limit necessary since the connection is flat.) By a real version of the flat index theorem [APS2], the difference of these bundles determines a class $x \in K O^{-1}(\mathcal{X} ; \mathbb{Z} / 2 \mathbb{Z})$, and the ratio of the holonomies is $\pi_{!}^{\mathcal{X}}(x) \in K O^{-4}(\mathrm{pt} ; \mathbb{Z} / 2 \mathbb{Z}) \cong \mathbb{Z} / 2 \mathbb{Z}$ (see (3.3)). As in previous arguments we use excision to localize the computation to $(I, \partial I) \times \partial \mathcal{X}$, where we write $x$ as the product of $[\eta-1] \in K O^{-1}((I, \partial I) ; \mathbb{Z} / 2 \mathbb{Z})$ and $\left[F^{\prime}\right] \in K O(\partial \mathcal{X})$. Thus

$$
\pi_{!}^{\mathcal{X}}(x)=\pi_{!}^{(I, \partial I)}([\eta-1]) \cdot \pi_{!}^{\partial \mathcal{X}}\left(\left[F^{\prime}\right]\right) \in \mathbb{Z} / 2 \mathbb{Z} .
$$

The first factor is 1 and the second is computed in $(5.23)-(5.24)$ to be $(\partial \phi)^{*} w_{2}(\nu)[\partial \mathcal{X}]$, as desired.

We proceed to the second step of the proof of Theorem 5.5, which is the proof of Proposition 5.10. We demonstrate that $L_{\gamma}^{+}$is trivializable geometrically, that is, as a bundle with metric and connection.

Proof of Proposition 5.10. Consider first the case when $\alpha=\beta$ and $\theta$ is the identity map. Then the orientation-reversing involution of the double manifold lifts to the spin bundle. In this case we claim that each of the three factors in (4.2) is trivializable. First, consider a family of surfaces with boundary $\pi: X \rightarrow Z$ and the associated family of doubles $\pi^{d}: X^{d} \rightarrow Z$. The determinant and pfaffian bundles are flat since the curvature (3.1) is the integral over the fibers of $\pi^{d}$ of a differential form which is invariant under the orientation-reversing involution on the double, so vanishes. Next, we investigate the holonomy. Consider a family of surfaces with boundary $\pi: X \rightarrow S^{1}$ and the associated double $\pi^{d}: X^{d} \rightarrow S^{1}$. We endow $S^{1}$ with the bounding spin structure and lift to a spin structure on $X^{d}$. The holonomy of the first factor of (4.2) is computed by a certain $\tau^{-1 / 2}$-invariant and that of each of the last two factors by a $\tau$-invariant. Since $X^{d}$ is the spin double of $X$, we compute these invariants using (3.5). Thus in each case the invariant is $(-1)^{\left(\begin{array}{c}k \\ 2\end{array}\right)}$, where $k$ is the number of components of $\partial X$ with a nontrivial index (or mod 2 index) of the appropriate boundary Dirac operator. For the last factor the operator on any component of the boundary is the boundary of a family of operators on the disk, so the index vanishes. For the first factor we note that the bundle $(\partial \phi)^{*}(T Y) \rightarrow \partial X$ is trivializable, since it is oriented and spin, and now since the base $S^{1}$ bounds a disk the mod 2 index vanishes. A similar argument applies to the second factor: rewrite the determinant line bundle as a pfaffian line bundle, as in (4.10). 
For general $\alpha, \beta, \theta$ the result now follows from Theorem 4.5.

This completes the proofs of Theorem 5.5 and Theorem 5.6.

\section{Additional Remarks.}

\section{The A- and B-Fields}

Fields which are locally differential forms often have a nontrivial global structure. This was explained a bit in $\S 1$; here we add a few details. Mathematical foundations for the low degree case needed here are developed in [B], though we do not use that language. ${ }^{12}$ For a related exposition, see $[\mathrm{DeF}, \S 6]$.

We distinguish four types of $p$-form fields, two types with nontrivial global structure together with their field strengths. For $p$ small we can say what they are in familiar geometric language:

\begin{tabular}{|c|c|c|c|}
\hline notation & $p$ & global description & global $p$-form? \\
\hline$\Theta^{1}$ & 1 & connection on principal T-bundle & no \\
\hline$\Omega_{\mathbb{Z}}^{2}$ & 2 & curvature of connection & yes \\
\hline$\Gamma^{0}$ & 0 & $\begin{array}{c}\text { section of principal } \mathbb{T} \text {-bundle } \\
\text { with connection }\end{array}$ & no \\
\hline$\Omega^{1}$ & 1 & covariant derivative of section & yes \\
\hline
\end{tabular}

A principal circle $(\mathbb{T})$ bundle over a manifold $M$ is a manifold $P$ on which $\mathbb{T}$ acts freely with quotient $M$. The bundle $P \rightarrow M$ is classified topologically by its first Chern class in integral degree two cohomology. Equivalently, we can view $P$ as the set of unit vectors in a hermitian line bundle over $M$. A connection is an imaginary 1-form on $P$ which satisfies some affine equations--it is not a differential form on the base $M$. The notion that a 1-form in field theory is often such a connection is quite familiar. The curvature is a closed 2-form on the base $M$ whose periods are integer multiples of $2 \pi i$. A section, or trivialization, of $P$ is a map $M \rightarrow P$ which splits the projection $P \rightarrow M$. Equivalently, it is a unit norm section of the associated hermitian line bundle. In general such sections exist locally; the Chern class of $P$ is an obstruction to global existence. The covariant derivative of a section is the pullback of the connection form to $M$, a global 1-form which is not necessarily closed.

The analog of the first two lines in lower degree may also be stated in familiar terms:

\begin{tabular}{|c|c|c|c|}
\hline notation & $p$ & global description & global $p$-form? \\
\hline$\Theta^{0}$ & 0 & map to $\mathbb{T}$ & no \\
\hline$\Omega_{\mathbb{Z}}^{1}$ & 1 & $\log$ derivative of map & yes \\
\hline
\end{tabular}

An object in the first line is a map $g: M \rightarrow \mathbb{T}$. The corresponding field strength $d \log g$ is a global closed 1-form on $M$ whose periods are integer multiples of $2 \pi i$. Note that (6.2) is the special case of the last two lines of (6.1) when the circle bundle with connection is trivial and trivialized.

${ }^{12}$ As remarked in the introduction, a non-Čech mathematical theory adequate for all examples in string theory and $M$-theory is lacking. 
The $B$-field on spacetime $Y$ is an element of $\Theta^{2}(Y)$. In other words, it is the $p=2$ analog of the first line of (6.1) and (6.2). Topologically, it is classified by a characteristic class $\zeta_{B} \in H^{3}(Y ; \mathbb{Z})$, analogous to the Chern class of a circle bundle in integral $H^{2}$. Geometrically, we describe $B$ in terms of an open cover $\left\{U_{i}\right\}$ of $Y$. On each open set $U_{i}$ there is a 2 -form $B_{i} \in \Omega^{2}\left(Y_{i}\right)$, but on overlaps $U_{i j}=U_{i} \cap U_{j}$ they do not necessarily agree. Rather, there is a 1 -form $\alpha_{i j} \in \Omega^{1}\left(U_{i j}\right)$ such that

$$
B_{i}-B_{j}=d \alpha_{i j} \quad \text { on } U_{i j}
$$

Similarly, on the triple intersection $U_{i j k}=U_{i} \cap U_{j} \cap U_{k}$ there is given a circle-valued function $g_{i j k}: U_{i j k} \rightarrow \mathbb{T}$ such that

$$
d \log g_{i j k}=\exp \left[\sqrt{-1}\left(\alpha_{j k}-\alpha_{i k}+\alpha_{i j}\right)\right] \quad \text { on } U_{i j k} .
$$

The $g_{i j k}$ satisfy the cocycle relation

$$
g_{j k \ell} g_{i k \ell}^{-1} g_{i j \ell} g_{i j k}^{-1}=1 \quad \text { on } U_{i j k \ell} \text {. }
$$

The $B$-field is the triple $B=\left\{B_{i}, \alpha_{i j}, g_{i j k}\right\}$, which may be conveniently placed in a double complex:

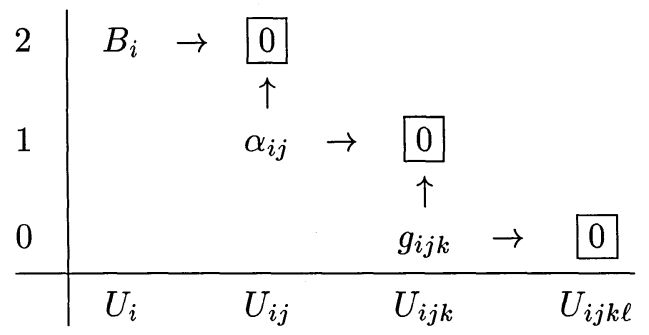

Note $d B_{i}=d B_{j}=H$ is a global 3 -form which is closed with $2 \pi i \mathbb{Z}$ periods; it is the field strength of the $B$-field. The boxes around the three zeros in (6.6) are shorthand for equations (6.3)-(6.5). The horizontal arrows denote the Cech differential and the vertical arrows denote plus or minus the de Rham differential $d$.

In the bosonic string with a $D$-brane, the $A$-field on the brane $\iota: Q \hookrightarrow Y$ is an element of $\Gamma^{1}(Q)$, that is, a generalization of the third line of (6.1) to $p=1$. However, it is related to the restriction of the $B$-field to the brane in a specific way. Namely, in terms of the restriction of the covering $\left\{U_{i}\right\}$ to $Q$, we write $A=\left\{A_{i}, h_{i j}\right\}$ in the diagram:

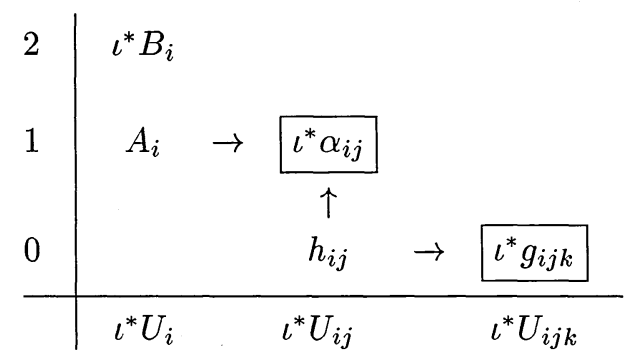

We impose equations at the two boxes. Specifically,

$$
\begin{aligned}
A_{j}-A_{i}-d \log h_{i j} & =\iota^{*} \alpha_{i j} & & \text { on } \iota^{*} U_{i j} \\
h_{j k} h_{i k}^{-1} h_{i j} & =\iota^{*} g_{i j k} & & \text { on } \iota^{*} U_{i j k} .
\end{aligned}
$$


We make two remarks about these equations. First, if $B=0$ so that the right hand sides of (6.8) vanish, then $\left\{h_{i j}\right\}$ are the transition functions of a circle bundle and $\left\{A_{i}\right\}$ patch to a connection on it. In other words, if $B=0$ then the $A$-field is a connection on a circle bundle-an element of $\Theta^{1}(Q)$ as in the first line of (6.1). This is the usual interpretation of the gauge field on the brane. Second, for general $B$ fields we can say that $A$ is a "trivialization" of the restriction $\iota^{*} B$, where the precise meaning of "trivialization" is (6.8). By analogy consider the case one degree lower, where $B$ is a circle bundle with connection and $A$ a trivialization. There are two possible meanings to "trivialization" in this context: topological and geometric. A topological trivialization corresponds to the second equation of (6.8) only. A geometric trivialization corresponds to imposing three equations- the equations of (6.8) plus the equation $d A_{i}=\iota^{*} B_{i}$. The intermediate case of two equations which we use has no analog one degree lower. Note that in our case the difference $d A_{i}-\iota^{*} B_{i}$ is a global 2 -form which is not necessarily closed. It is the analog of the covariant derivative in the fourth line of (6.1). For $B=0$ it is the curvature of the abelian gauge field $A$.

Now we make contact with the discussion in $\S 1$. For the bosonic string the term (1.10) in the action is a well-defined number for each field configuration. One degree down, where $B$ is a connection on a circle bundle and $A$ a trivialization of $\iota^{*} B$, this term corresponds to the parallel transport along a path viewed as a number using the trivialization on the boundary. Hence this part of the action is well-defined as a function-not a section of a line bundle-so does not contribute to the anomaly.

In the superstring what was explained in $\S 1$ is that since the first factor in (1.11) is a section of a nontrivial line bundle (over the space of metrics on $\Sigma$ and maps $\Sigma \rightarrow Y$ ), we must modify the global interpretation of the $A$-field in order that the product of the last two factors be a section of the inverse line bundle with connection. In our present context we describe the modification as follows. First, the second Stiefel-Whitney class $w_{2}(\nu)$ of the normal bundle $\nu$ to $Q$ in $Y$ determines a flat $B_{w_{2}(\nu)} \in \Theta^{2}(Q)$, defined in (1.13). In terms of the open covering $\left\{\iota^{*} U_{i}\right\}$ it can be written as

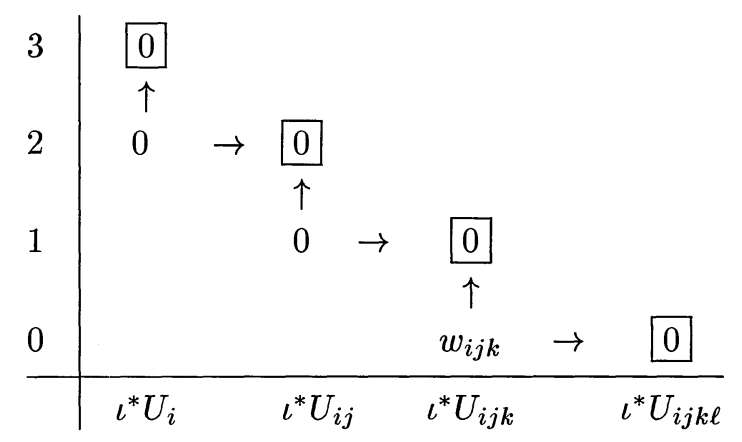

where $\left\{w_{i j k}= \pm 1\right\}$ is a Cech cocycle for $w_{2}(\nu)$. Then the $A$-field is an isomorphism

$$
A: B_{w_{2}(\nu)} \longrightarrow \iota^{*} B
$$

where "isomorphism" is understood in the sense of equations (6.8), as explained above. 
In other words, $A=\left\{A_{i}, h_{i j}\right\}$ fits into a slight modification of (6.7):

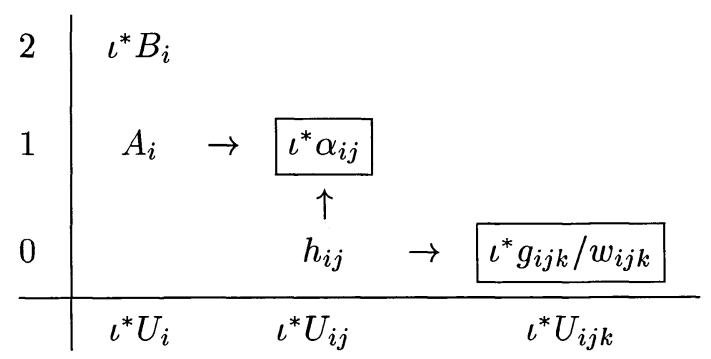

With this $A$-field (1.10) is no longer a function, but rather a section of a flat line bundle with connection over the space of parameters. The holonomy of that bundle is computed by integrating $-w_{2}(\nu)=w_{2}(\nu)$ over the boundary of the surface mapping in, and this precisely cancels the line bundle with connection from the fermionic determinants (as computed in Theorem 5.6). Thus the product of terms in (1.11) is a section of a trivializable line bundle with connection: there is no anomaly.

The existence of an isomorphism in the sense of equations (6.8) is equivalent to the existence of a topological isomorphism, which only exists if

$$
\iota^{*} \zeta_{B}=W_{3}(\nu)
$$

where $\zeta_{B} \in H^{3}(Y ; \mathbb{Z})$ is the topological characteristic class of the $B$-field and $W_{3}(\nu)$ is the third Stiefel-Whitney class of the normal bundle. Equation (6.9), which is the same as (1.12), is a topological restriction on branes which may occur in a spacetime $Y$ with given $B$-field. It was first discovered in a nonperturbative setting in [W3]; the present paper derives (6.9) from the perturbative string.

Note in particular that if $B=0$, then (6.9) asserts that $\nu$ admits a Spin ${ }^{c}$ structure. In that case the $A$-field, which trivializes $B_{w_{2}(\nu)}$ in the sense we described here, is a $\operatorname{Spin}^{c}$ connection. (The reader should relate our Cech description with other definitions of $\operatorname{Spin}^{c}$ connections.)

\section{Dirac Operators with Local Boundary Conditions ${ }^{13}$}

Immediately following the first proof of the index theorem on closed manifolds, Atiyah, Bott, and Singer $[\mathrm{AB}]$ proved a topological index theorem for general elliptic operators with local boundary conditions. They observed that there is a topological obstruction to the existence of local boundary conditions, and that local boundary conditions, when they exist, lift the symbol class of the operator in $K$-theory to a relative class. It is in terms of this lifted class that one obtains a topological index formula. Later, Atiyah, Patodi, and Singer [APS1] introduced global boundary conditions for first-order Dirac operators, and these always exist. Perhaps for that reason it is global, rather than local, boundary conditions which appear in most of the index theory literature. In this subsection we generalize the local boundary conditions discussed in $\S 5$ to Dirac operators in arbitrary even dimensions, and then we indicate how geometric aspects of index theory may be treated by doubling. (Doubling is a common technique in the theory of elliptic boundary-value problems in flat space as well, and it is also the main technique in the Atiyah-Bott paper.) We only indicate a

\footnotetext{
${ }^{13}$ We are indebted to Xianzhe Dai for discussions about the issues treated here.
} 
rough outline of the arguments; we have not carried through the details. For simplicity we deal only with complex Dirac operators, though the discussion applies to real Dirac operators as well.

As an aside, we remark that generally in physics boundary conditions play at least two different roles. An object (such as a $D$-brane or the Earth's ocean) may have a boundary, at which one imposes local boundary conditions. The normal vector to such a boundary is ordinarily spacelike. On the other hand, an important physical and mathematical technique is to "cut" on a spacelike surface to reveal a quantum state. The normal vector to such a boundary is generally timelike if we work with Lorentz signature, and otherwise spacelike. On such a cut, one uses global boundary conditions, similar to those used in index theory in factorization theorems, such as the one leading to (3.5).

Consider an even-dimensional $\operatorname{Spin}^{c}$ manifold $X$ with boundary. Let $S^{ \pm}$denote the spinor bundles on $X$. Let $\zeta$ be the unit outward normal vector field at the boundary. The Clifford multiplication

$$
c(\zeta):\left.\left.S^{+}\right|_{\partial X} \longrightarrow S^{-}\right|_{\partial X}
$$

is an isomorphism. Suppose $E^{ \pm} \rightarrow X$ are complex vector bundles (with connection) and we are given an isomorphism

$$
\tau:\left.\left.E^{+}\right|_{\partial X} \longrightarrow E^{-}\right|_{\partial X}
$$

at the boundary. Then the Dirac operator

$$
D^{X}: S^{+} \otimes E^{+} \oplus S^{-} \otimes E^{-} \longrightarrow S^{-} \otimes E^{+} \oplus S^{+} \otimes E^{-}
$$

admits the local boundary condition

$$
(c(\zeta) \otimes \tau)\left(\left.\psi^{+}\right|_{\partial X}\right)=\left.\psi^{-}\right|_{\partial X}
$$

on a pair $\left(\psi^{+}, \psi^{-}\right)$of sections of $\left(S^{+} \otimes E^{+}, S^{-} \otimes E^{-}\right)$. The boundary-value problems considered in section 5 are special cases. Note that different spin (or $\operatorname{spin}^{c}$ ) structures on $S^{+}, S^{-}$may be accommodated by tensoring $E^{-}$with a real (or complex) line bundle. We propose that this class of Dirac operators has a good geometric index theory, analogous to that of Dirac operators on closed manifolds.

It is not hard to check that (6.12) defines elliptic boundary conditions (as described in $[\mathrm{AB}]$, for example). Therefore, the Dirac operator enjoys the same basic analytic properties as those of a Dirac operator on a closed manifold: the spectrum is discrete, there is a meromorphic $\zeta$-function, etc. There is no problem carrying out geometric constructions, such as the determinant line bundle with metric and connection, in families of such operators. From such constructions one will obtain formulas for geometric invariants, such as curvature and holonomy of the determinant line bundle, directly on $X$. In the remainder of this section we indicate how to reduce these constructions to the closed case by doubling.

We restrict to manifolds $X$ whose Riemannian metric is a product near the boundary. Let $X^{d}$ denote the smooth closed Riemannian manifold obtained by gluing $X$ to $-X$ along $\partial X$. We glue $S^{+} \rightarrow X$ and $S^{-} \rightarrow-X$ using (6.10) to obtain the plus spinor bundle $S_{X^{d}}^{+} \rightarrow X^{d}$, and similarly glue $E^{+} \rightarrow X$ to $E^{-} \rightarrow-X$ to form a complex vector bundle $E \rightarrow X^{d}$ (with connection). Our goal is to precisely relate the boundary-value problem (6.11), (6.12) with the Dirac operator $D_{X^{d}}^{+}(E)$ on the double. We adapt the gluing argument of $[\mathrm{DF}, \S \mathrm{IV}]$, which we summarize in Figures 1 and 2. 

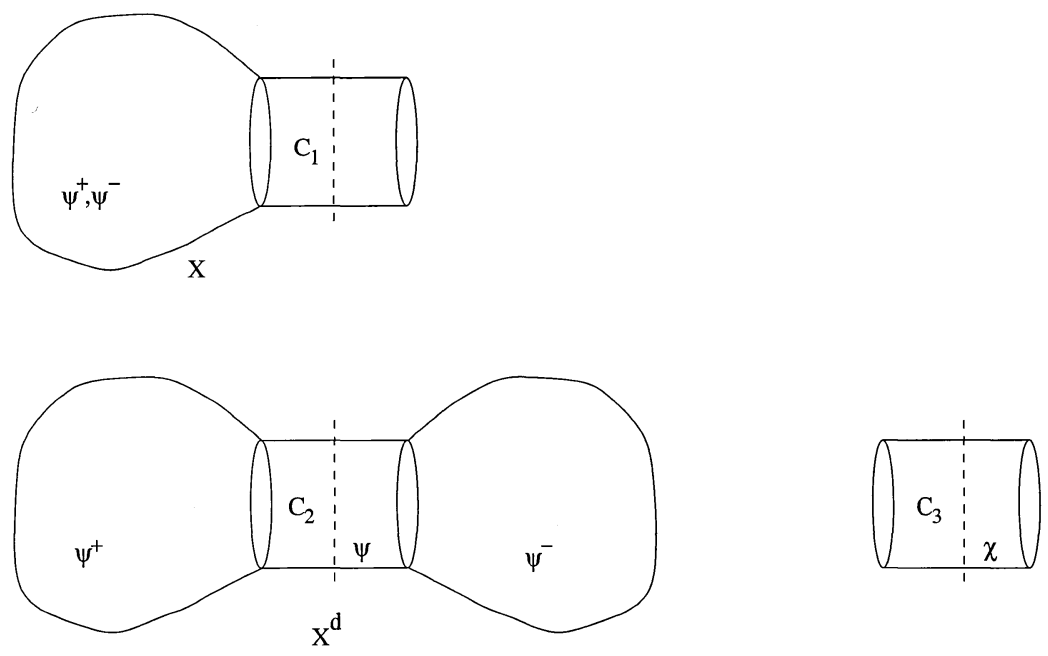

Figure 1: Gluing SPINOR FieldS

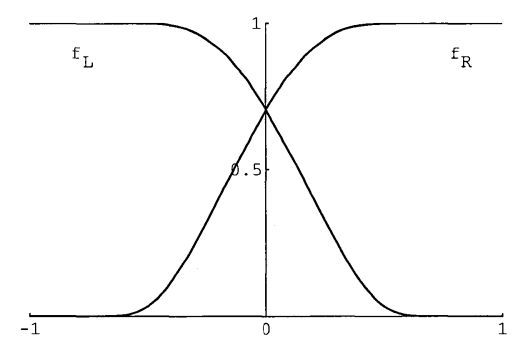

Figure 2: Cutoff FUnCTIONS

Let $\Gamma$ denote the space of smooth sections, regarded as an inner product space using the $L^{2}$ metric. The figures summarize a gluing map

$$
U: \Gamma_{X}\left(S^{+} \otimes E^{+} \oplus S^{-} \otimes E^{-}\right) \longrightarrow \Gamma_{X^{d}}\left(S^{+} \otimes E\right) \oplus \Gamma_{C_{3}}\left(S^{+} \otimes E\right),
$$

which for appropriate cutoff functions is an isometry. The spinor fields $\left(\psi^{+}, \psi^{-}\right)$in the domain satisfy the boundary condition (6.12), and the spinor field $\chi$ on $C_{3}$ satisfies

$$
\chi(t=1)=-\chi(t=-1),
$$

where $t$ is the axial coordinate on the cylinder. More explicitly, the gluing map on cylinders is

$$
\left(\begin{array}{c}
\psi(t) \\
\chi(t)
\end{array}\right)=\left(\begin{array}{cc}
f_{L}(t) & f_{R}(t) \\
-f_{R}(t) & f_{L}(t)
\end{array}\right)\left(\begin{array}{c}
\psi^{+}(t) \\
\psi^{-}(-t)
\end{array}\right), \quad \psi^{+}, \psi^{-} \text {on } C_{1}, \quad \psi \text { on } C_{2}, \quad \chi \text { on } C_{3} .
$$

The cutoff functions $f_{L}, f_{R}:[-1,1] \rightarrow[0,1]$ satisfy

$$
\begin{aligned}
f_{L}([-1,-1 / 2]) & =f_{R}([1 / 2,1])=1 & f_{L}([1 / 2,1]) & =f_{R}([-1,-1 / 2])=0 \\
f_{L}^{2}+f_{R}^{2} & =1 & f_{L}(-x) & =f_{R}(x) .
\end{aligned}
$$


The same gluing works in a family of operators; the result is an isometry of infinite dimensional vector bundles of spinor fields.

The claim is that the determinant line bundle for the Dirac operator on $C_{3}$ is canonically trivial, and so, after some more argument, in such a family the determinant line bundle (with its metric and connection) for the boundary-value problem on $X$ is canonically isomorphic to the determinant line bundle for the closed manifold $X^{d}$.

So as not to rely on the details of this argument, which we may present elsewhere, in this paper we adapted the practical point of view of defining the metric and connection directly from the Dirac operator on the double.

\section{REFERENCES}

[A] J. F. Adams, Vector-fields on spheres, Bull. Amer. Math. Soc, 68 (1962), pp. 39-41.

[AB] M. F. AtiYAh AND R. Bott, The index problem for manifolds with boundary, in Differential Analysis, Bombay Colloq., 1964, Oxford Univ. Press, London, 1964, pp. 175-186.

[APS1] M. F. AtiYah, V. K. Patodi, ANd I. M. Singer, Spectral asymmetry and Riemannian geometry. I, Math. Proc. Cambridge Philos. Soc., 77 (1975), pp. 43-69.

[APS2] M. F. AtiYah, V. K. Patodi, ANd I. M. Singer, Spectral asymmetry and Riemannian geometry. III, Math. Proc. Cambridge Philos. Soc., 79 (1976), pp. 71-99.

[AS1] M. F. AtiYah AND I. M. Singer, The index of elliptic operators III, Ann. of Math., 87 (1968), pp. 546-604.

[AS2] M. F. AtiYAH AND I. M. Singer, The index of elliptic operators V, Ann. of Math., 93 (1971), pp. 139-149.

[B] J.-L. BRYLINSKI, Loop spaces, characteristic classes and geometric quantization, Progr. Math., 107, Birkhäuser, Boston, MA, 1993.

[BF] J. M. Bismut And D. S. Freed, The analysis of elliptic families I: Metrics and connections on determinant bundles, Commun. Math. Phys., 106 (1986), pp. 159-176; II: Dirac operators, eta invariants, and the holonomy theorem of Witten, Commun. Math. Phys., 107 (1986), pp. 103-163.

[BW] B. Booss-Bavnbek And K. P. Wojciechowski, Elliptic Boundary Problems for Dirac Operators, Birkhäuser, Boston, 1993.

[BH] A. Borel And F. Hirzebruch, Characteristic classes and homogeneous spaces. II, Amer. J. Math., 81 (1959), pp. 315-382.

[CY] Y.-K. E. Cheung And Z. YIn,, Anomalies, Branes, and Currents, Nucl. Phys., B517 (1998), pp. 185-196, hep-th/9803931.

[DF] X. Dai And D. S. Freed, $\eta$-invariants and determinant lines, J. Math. Phys., 35 (1994), pp. $5155-5194$.

[DZ] X. Dai and W. Zhang, Splitting of the family index, Commun. Math. Phys., 182 (1996), pp. 303-317.

[DeF] P. Deligne and D. S. Freed, Classical field theory, in Quantum Fields and Strings: A Course for Mathematicians, Volume 1, P. Deligne, P. Etingof, D. S. Freed, L. C. Jeffrey, D. Kazhdan, J. W. Morgan, D. R. Morrison, E. Witten, eds., American Mathematical Society, Providence, RI, 1999, pp. 137-225.

[F1] D. S. FreED, On determinant line bundles, in Mathematical Aspects of String Theory, ed. S. T. Yau, World Scientific Publishing, 1987.

[F2] D. S. Freed, Determinants, torsion, and strings, Commun. Math. Phys, 107 (1986), pp. 483513.

[F3] D. S. Freed, Two index theorems in odd dimensions, Commun. Anal. Geom., 6 (1998), pp. 317-329, dg-ga/9601005.

[FM] D. S. Freed and J. W. Morgan, Appendix to [F2], Commun. Math. Phys, 107 (1986), pp. 510-513.

[GHM] M. Green, J. A. Harvey, And G. Moore, I-Brane Inflow And Anomalous Couplings On D-Branes, Class. Quant. Grav., 14 (1997), pp. 47-52, hep-th/9605033.

[H] P. Horava, Type IIA D-Branes, K Theory, And Matrix Theory, Adv. Theor. Math. Phys., 
2 (1999), pp. 1373-1404, hep-th/9812135.

[MM] G. Moore and R. Minasian, K Theory And Ramond-Ramond Charge, JHEP 9711:002, 1997, hep-th/9710230.

[N] L. I. NiCOLAESCU, Generalized symplectic geometries and the index of families of elliptic problems, Mem. Amer. Math. Soc., 128:609 (1997).

[P] P. PIAzza, Determinant bundles, manifolds with boundary and surgery, Commun. Math. Phys., 178 (1996), pp. 597-626; II. Spectral sections and surgery rules for anomalies, Commun. Math. Phys., 193 (1998), pp. 105-124.

[S] A. SEn, Tachyon Condensation On The Brane Antibrane System, JHEP 9808:012, 1998, hep-th/9805170.

[W1] E. Witten, Global gravitational anomalies, Commun. Math. Phys., 100 (1985), pp. $197-229$.

[W2] E. Witten, Global anomalies in string theory, in Anomalies, Geometry, and Topology, edited by A. White, World Scientific, 1985, pp. 61-99.

[W3] E. WitTen, Baryons And Branes In Anti-de Sitter Space, JHEP 9807:006, 1998, hepth/9805112.

[W4] E. Witten, D-Branes And K-Theory, JHEP 9812:025, 1998, hep-th/9810188. 
